Published by Cambridge University Press

This is an Open Access article, distributed under the terms of the Creative Commons

Attribution-NonCommercial-NoDerivatives licence (http://creativecommons.org/licenses/by-nc-nd/4.0/), which permits non-commercial re-use, distribution, and reproduction in any medium, provided the original work is unaltered and is properly cited. The written permission of Cambridge University Press must be obtained for commercial re-use or in order to create a derivative work.

doi:10.1017/S0022377820001117

\title{
Divertor heat flux challenge and mitigation in SPARC
}

\author{
A. Q. Kuang ${ }^{\circledR 1}{ }^{\dagger}$, S. Ballinger ${ }^{1}$, D. Brunner ${ }^{2}$, J. Canik ${ }^{3}$, A. J. Creely ${ }^{\circledR 2}$, \\ T. Gray ${ }^{3}$, M. Greenwald ${ }^{\circledR 1}$, J. W. Hughes ${ }^{\circledR 1}$, J. Irby ${ }^{1}$, \\ B. LaBombard ${ }^{1}$, B. Lipschultz ${ }^{4}$, J. D. Lore $^{3}$, M. L. Reinke ${ }^{3}$, J. L. Terry ${ }^{1}$, \\ M. Umansky ${ }^{5}$, D. G. Whyte ${ }^{1}$, S. Wukitch ${ }^{1}$ and the SPARC Team \\ ${ }^{1}$ Plasma Science and Fusion Center, MIT, Cambridge, MA 02139, USA \\ ${ }^{2}$ Commonwealth Fusion Systems, Cambridge, MA 02139, USA \\ ${ }^{3}$ Oak Ridge National Laboratory, Oak Ridge, TN 37831, USA \\ ${ }^{4}$ York Plasma Institute, University of York, Heslington, York YO10 5DD, UK \\ ${ }^{5}$ Lawrence Livermore National Laboratory, Livermore, CA 94550, USA
}

(Received 13 June 2020; revised 23 August 2020; accepted 1 September 2020)

Owing to its high magnetic field, high power, and compact size, the SPARC experiment will operate with divertor conditions at or above those expected in reactor-class tokamaks. Power exhaust at this scale remains one of the key challenges for practical fusion energy. Based on empirical scalings, the peak unmitigated divertor parallel heat flux is projected to be greater than $10 \mathrm{GW} \mathrm{m}^{-2}$. This is nearly an order of magnitude higher than has been demonstrated to date. Furthermore, the divertor parallel Edge-Localized Mode (ELM) energy fluence projections $\left(\sim 11-34 \mathrm{MJ} \mathrm{m}^{-2}\right)$ are comparable with those for ITER. However, the relatively short pulse length $(\sim 25 \mathrm{~s}$ pulse, with a $\sim 10 \mathrm{~s}$ flat top $)$ provides the opportunity to consider mitigation schemes unsuited to long-pulse devices including ITER and reactors. The baseline scenario for SPARC employs a $\sim 1 \mathrm{~Hz}$ strike point sweep to spread the heat flux over a large divertor target surface area to keep tile surface temperatures within tolerable levels without the use of active divertor cooling systems. In addition, SPARC operation presents a unique opportunity to study divertor heat exhaust mitigation at reactor-level plasma densities and power fluxes. Not only will SPARC test the limits of current experimental scalings and serve for benchmarking theoretical models in reactor regimes, it is also being designed to enable the assessment of long-legged and X-point target advanced divertor magnetic configurations. Experimental results from SPARC will be crucial to reducing risk for a fusion pilot plant divertor design.

Key words: plasma devices, fusion plasma

$†$ Email address for correspondence: aqkuang@mit.edu 


\section{Introduction}

The interface/interaction between the high plasma power/particle fluxes and plasma-facing components (PFCs) in the presence of reactor-level power flows has long been recognized as a critical issue for fusion energy ('Report on Science Challenges and Research Opportunities in Plasma Materials Interactions', US Department of Energy 2015). SPARC, a compact, high-magnetic-field tokamak, aiming to demonstrate net energy from fusion (Creely et al. 2020), will be prototypical of divertor power exhaust requirements. Empirical scalings (Eich et al. 2013; Brunner et al. 2018b; Eich et al. 2020) for the scrape-off layer (SOL) heat flux width, indicate that unprecedented high unmitigated peak parallel heat fluxes $\left(q_{\|}>10 \mathrm{GW} \mathrm{m}^{-2}\right)$ are anticipated in the SPARC divertor. Furthermore, transient events such as edge-localized modes (ELMs) and disruptions impose heat flux and structural loading conditions comparable with ITER and will pose a significant challenge for SPARC (Hughes et al. 2020; Sweeney et al. 2020). At the same time, it is important to note that these predictions for SPARC rely on extrapolations from the experimental databases. Measurements in SPARC would be valuable for benchmarking these experimental scalings as well as validating first principles computational models, for example XGC (Chang et al. 2017), for reactor conditions for the first time, thus increasing our confidence in subsequent predictions for ITER and a future fusion pilot plant such as ARC (Sorbom et al. 2015).

In present-day pulsed devices, heat exhaust management is achieved by keeping heat fluxes and pulse lengths at levels that are low relative to material limits and/or employing engineering designs with intra-shot cooling. In some high-power cases, for example in JET (Telesca et al. 2017) and ASDEX-Upgrade (Kallenbach et al. 2015), control of heat flux to PFCs is necessary and low- $Z$ divertor impurity seeding is needed to access dissipative divertor regimes. How these impurity seeding experiments might scale to the SPARC device and its effect on the ability to achieve the primary mission goal of $Q>2$ is unclear because of the sizable extrapolation to SPARC divertor parameters from these devices. Alternatively, active feedback control systems have also been developed that can reduce the heat flux to the divertor target surface without negatively affecting core plasma performance (Kallenbach et al. 2012; Brunner et al. 2017; Guillemaut et al. 2017; Courtois et al. 2019). Whether any of these active-feedback systems can be implemented in the SPARC device has yet to be assessed, but it will be challenging given the available diagnostic access in the nuclear environment and the actuator response times. As we cannot ensure, from a physics and control perspective, that SPARC will achieve a dissipative divertor in its net-energy operation, attached divertor conditions have to be assumed in the design of the SPARC divertor.

Similarly, although the SPARC magnets and PFCs are being designed to be up-down symmetric and the primary reference discharges are all double-null equilibria, there are concerns about the ability to control and maintain double null to the precision needed for power sharing across the upper and lower sets of divertors (De Temmerman et al. 2011; Brunner et al. 2018a). Hence, the baseline design scenario will also have to assume power-sharing fractions consistent with a single-null equilibrium. To avoid melting or sublimating of the inertially cooled divertor, the strike point will be swept at $\sim 1 \mathrm{~Hz}$ over the course of the $10 \mathrm{~s}$ discharge flat top, with the full sweep extent for SPARC (presently at a 'V2' design) shown in figure 1(a). Strike point sweeping for heat flux mitigation was a routine part of high-power operations on Alcator C-Mod (Reinke et al. 2019) and JET (Silburn et al. 2017).

With those conservative assumptions as a baseline, SPARC is also being designed to maximize the possibilities of accessing high-power dissipative divertor regimes. Simulations of SPARC V2 are being developed using UEDGE (Rognlien et al. 1992) 

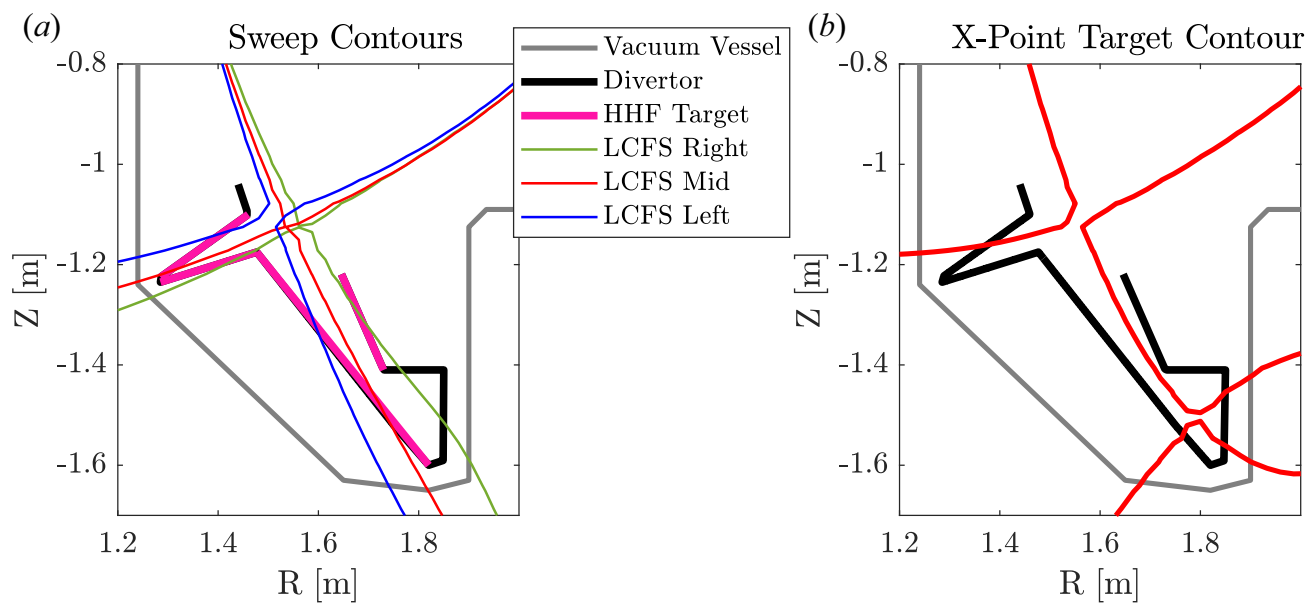

FIGURE 1. SPARC V2 equilibria: (a) the full extent of the strike point sweep equilibria with the high heat flux (HHF) surface of the divertor target highlighted in pink and $(b)$ the XPT configuration equilibrium.

and SOLPS-ITER (Wiesen et al. 2015; Bonnin et al. 2016). The simulation results are being used to bound divertor performance expectations, inform design requirements for the divertor control systems (e.g. gas injection and sweeping) and, finally, to predict wall particle and power fluxes on main chamber and divertor surfaces. Divertor heat flux management will be a key focus as the tokamak is commissioned to full power operations. Even with the limited diagnostics expected to be available, SPARC will uniquely open up new insights into dissipative divertor access and controllability at reactor level power and particle fluxes.

SPARC will be an important testbed for studying reactor-level divertor power and particle densities and the potential solutions for a future fusion pilot plant. Looking beyond the techniques used for high-powered discharges today, impurity injection and divertor neutral pressure control, SPARC will have a tightly baffled divertor for high neutral compression and inclined target plates to assist access to dissipative divertor regimes. Furthermore, 'advanced divertor' magnetic geometries present an interesting opportunity for SPARC to reduce heat loads, enhance access to detachment and improve detachment control. Therefore, the SPARC magnet coil set is being designed to produce a sub-class of 'advanced divertors', including long-legged and X-point target (XPT) magnetic geometries (figure $1 b$ ). The XPT magnetic geometry was first proposed as part of the ADX divertor test tokamak (LaBombard et al. 2015) design process and has since been incorporated into the ARC pilot plant design (Kuang et al. 2018). Although the XPT geometry can only be achieved in SPARC at reduced core plasma current, $I_{p}<5.7 \mathrm{MA}$, the core scenarios still project to a fusion power of up to $37 \mathrm{MW}$ and $Q \sim 2$ (Creely et al. 2020). SPARC will be a unique and useful platform for both answering key questions about the physics of such advanced divertor geometries and providing data that would be crucial for designing the divertor for ARC. The limitation on what questions can be answered and to what precision will be dictated by the divertor diagnostic set, which is currently under consideration.

This paper is focused on the ongoing characterization and assessment of the current SPARC divertor design. The paper is organized as follows: $\$ 2$ outlines the design guidelines for the SPARC divertor based on projections for SPARC derived from current empirical and theoretical models; $\S 3$ presents simulations of the divertor surface material 
temperature response to a strike point sweep, the conservative and primary heat flux mitigation scenario for the SPARC divertor if enough divertor power dissipation is not achieved; $\S 4$ introduces the development of next step models being developed to assess the access to dissipative divertor regimes and assist in refining the design guidelines; $§ 5$ provides a preliminary outline of the divertor XPT scenario being explored for SPARC; and, lastly, §6 discusses the uncertainty in the design guidelines resulting from the significant extrapolation of present devices to SPARC and highlights the potential for SPARC to be able to provide valuable data to increase the confidence in the design of a future fusion pilot plant.

\section{Divertor design guidelines for SPARC}

The empirical and heuristic models that have been developed to date are used to develop the heat loading design scenarios for the SPARC V2 divertor. There is a sizeable uncertainty in trying to determine the divertor target surface heat fluxes in a new device, especially because it requires extrapolation of present-day datasets. Therefore, conservative assumptions are used to ensure that SPARC can survive to achieve its primary scientific missions of $Q>2$.

Full field (12.2 T), full current (8.7 MA), DT H-mode discharges in SPARC V2 (Creely et al. 2020) are the reference discharge for designing the divertor. Based on the multi-machine scalings by Eich et al. (2013) for the steady-state heat flux width $\left(\lambda_{q}\right)$ under attached $\mathrm{H}$-mode conditions, the parallel heat flux entering into the divertor is proportional to $P_{\mathrm{SOL}} B_{0} / R_{0}$ (LaBombard et al. 2015), where $P_{\mathrm{SOL}}$ is defined as the conducted power crossing into the SOL, $B_{0}$ is the on-axis magnetic field and $R_{0}$ is the major radius. Values of $P_{\mathrm{SOL}} B_{0} / R_{0} \sim 30 \mathrm{MW} \cdot \mathrm{T} \mathrm{m}^{-1}$ have been achieved on Alcator C-Mod (Hughes et al. 2018) and ASDEX-Upgrade (Kallenbach et al. 2015). In preparation for DTE2, JET has also started to approach $\sim 31 \mathrm{MW} \cdot \mathrm{T} \mathrm{m}^{-1}$, assuming $0 \%$ core radiation (Garzotti et al. 2019). For the SPARC V2 full field, full current, DT H-mode, the normalized power of $P_{\mathrm{SOL}} B_{0} / R_{0} \sim 191 \mathrm{MW} \cdot \mathrm{T} \mathrm{m}^{-1}$ (assuming $39 \%$ core radiation fraction) is, thus, significantly higher than in present-day devices and approaches levels for proposed fusion pilot plants, such as $\mathrm{ARC}$, where $P_{\mathrm{SOL}} B_{0} / R_{0} \sim 256 \mathrm{MW} \cdot \mathrm{T} \mathrm{m}^{-1}$ (assuming the nominal $35 \%$ core radiation fraction) (Kuang et al. 2018). The scaling developed by Eich et al. (2013) is for the period between ELMs and attached divertor H-modes only. Although the full field L-mode discharge (Creely et al. 2020) has a higher projected value of $P_{\mathrm{SOL}}$ owing to the lower core radiation fraction, the wider $\lambda_{q}$ projected for SPARC L-mode relative to H-modes (Brunner et al. 2018b) at the same engineering parameters (e.g. poloidal magnetic field and $P_{\mathrm{SOL}}$ ) would result in a reduced parallel heat flux, relative to H-mode, entering into the divertor. Therefore, unless otherwise stated, for the rest of this paper the full field $\mathrm{H}$-mode reference discharge parameters summarized in table 1 will be used for modelling purposes, whereas the magnetic equilibriums used in this paper were generated using the FreeGS Grad-Shafranov solver (Dudson 2019).

The decision for divertor target material has yet to be made, with a current focus of the analysis of tungsten or carbon. Important trade-offs are still being assessed. Carbon-based PFC materials are preferred from a divertor heat flux handling standpoint. Decades of experience have shown that sublimation of carbon-based, temperature limited PFC designs allow failures from unintended overheating to avoid permanent damage that affects future operations. Conversely, a high-Z PFC material such as tungsten could just crack or melt. Melted tungsten in a tokamak environment has been shown to exhibit a positive feedback loop where the melt layer will pool and protrude from the PFC surface (Coenen et al. 2015; Pitts et al. 2017), creating large leading edges that further concentrate plasma heat flux. The core plasma is also significantly less tolerant to high- $Z$ core impurities, small 


$\begin{array}{lccc}\text { Parameter } & \text { Symbol } & \text { Full field H-mode } & \text { Units } \\ \text { On-axis toroidal field } & B_{0} & 12.2 & \mathrm{~T} \\ \text { Core major radius } & R_{0} & 1.85 & \mathrm{~m} \\ \text { Core minor radius } & a & 0.57 & \mathrm{~m} \\ \text { Plasma current } & I_{P} & 8.7 & \mathrm{MA} \\ \text { Core plasma elongation } & \kappa_{\mathrm{sep}} & 1.97 & \\ \text { Core plasma triangularity } & \delta_{\mathrm{sep}} & 0.54 & \\ \text { Edge safety factor } & q^{*} & 3.05 & \\ \text { Current flattop period } & \Delta t_{\text {flattop }} & 10 & \mathrm{~s} \\ \text { Volume-averaged temperature } & T_{e} & 7.3 & \mathrm{keV}^{-3} \\ \text { Volume-averaged density } & n_{e} & 3.1 & 10^{20} \mathrm{~m}^{-3} \\ \text { Conducted power into the SOL } & P_{\mathrm{SOL}} & 29 & \mathrm{MW} \\ \text { Greenwald fraction } & f_{\mathrm{GW}} & 0.37 & \\ \text { Effective ion charge } & Z_{\mathrm{eff}} & 1.5 & \\ & & & \end{array}$

TABLE 1. Full field H-mode projections for SPARC V2. These are based on 1-D calculations by Creely et al. (2020).

quantities can easily lead to the radiative collapse of the plasma resulting in a disruption that will place significant structural and thermal loads on the SPARC device (Sweeney et al. 2020). However, carbon as a PFC material does not project towards a reactor due to the high erosion rates and tritium retention concerns (Andrew et al. 1999; Tanabe et al. 2003; Skinner \& Gianfranco 2006). Owing to the relatively short integrated discharge time over the course of the device lifetime, these issues do not immediately preclude the use of carbon PFCs in SPARC but they are nonetheless still a concern, e.g., the effect of dust production on high-power ICRF antenna operations, and is being carefully evaluated by the team. In this paper, the assumed PFC material for each analysis is specified.

\subsection{Divertor power exhaust}

The amount of conducted power crossing the last closed flux surface (LCFS) and entering the SOL is denoted as $P_{\mathrm{SOL}}$. It is calculated as the sum of power from auxiliary (11.1 MW), ohmic (1.7 MW) and alpha heating ( $20-28 \mathrm{MW})$ less the core radiated power $(\sim 10.4-13.2 \mathrm{MW})$. Estimates of core plasma impurity content assumed in zero-dimensional (0-D) calculations by Creely et al. (2020), predict $29 \mathrm{MW}$ of conducted power through the LCFS, whereas 1.5-D time-dependent core modelling simulations by Rodriguez-Fernandez et al. (2020), using different physics models and slightly different assumptions about impurities, yield $P_{\mathrm{SOL}}=19-21 \mathrm{MW}$, owing to reduced fusion power predictions and increased core radiation. Although the core impurity assumptions used are based on the existing tokamak database, they have wide uncertainties. Therefore, for the design of the divertor targets, $P_{\mathrm{SOL}}=29 \mathrm{MW}$, is assumed as the upper limit. Although some of the power flowing into the SOL will flow across SOL flux surfaces to the main chamber limiter surfaces, or radiated in the main chamber SOL, we ignore these loss channels and make the conservative assumption that all power goes to the divertors. As maintaining perfect double-null power sharing during a discharge will likely be challenging with the narrow SOL in SPARC, each set of divertors (upper and lower) will be designed to handle the full $P_{\text {SOL }}$ and no benefits from double-null power sharing is assumed. In single-null scenario ELM-suppressed EDA H-modes, the power sharing between the inner and outer divertor has been measured in Alcator C-Mod to be 
Scaling

Eich et al. (2013) Regression 14

Eich et al. (2013) Regression 15

Eich et al. (2020)

Goldston (2011)

Brunner et al. (2018b)
OMP heat flux width [mm]
0.18
0.3
0.43
0.4
0.35

TABLE 2. Projections for the heat flux width mapped to the OMP in SPARC V2. Note that for Eich et al. (2020), the dimensionally correct regression is used.

approximately $40: 60$ (Brunner et al. 2018a). In comparison, measurements of MAST for single-null $\mathrm{H}$-mode discharges with off-axis neutral beam heating recorded the inter-ELM integrated energy to the outer divertor to be 6.5 times higher than at the inner divertor. The ELM integrated energy deposition was found to be approximately three times higher on the outer divertor (De Temmerman et al. 2011). This suggests that the inter-ELM divertor IN:OUT sharing is approximately $15: 85$ and, for the ELMs, $25: 75$. Owing to the large uncertainty in these measurements, $40 \%$ of $P_{\mathrm{SOL}}$ is assumed to go to the inner divertor and $70 \%$ to the outer divertor, an additional $10 \%$ power has been included to provide some margin. Thus, the inner divertors on SPARC are being designed to exhaust 11.6 MW and the outer divertor $20.3 \mathrm{MW}$ for a $10 \mathrm{~s}$ pulse.

\subsection{SOL heat flux width}

To convert the power to the divertors into a surface heat flux, a key unknown quantity is the level of cross-field energy transport in the SOL. Without a validated model to make predictions, empirical and heuristic scalings for the heat flux e-folding width $\left(\lambda_{q}\right)$ are used for the design of SPARC. Table 2 summarizes the projections for the heat flux width mapped to the outer mid-plane (OMP) based on a set of recent scalings. The calculations are based on the parameters listed in table 1. However, some additional assumptions are required: the poloidal magnetic field at the outer midplane derived from the magnetic equilibrium of the primary reference discharge is $2.83 \mathrm{~T}$; the core plasma volume average ion temperature is assumed equal to $T_{e}$; the edge $Z_{\text {eff }}$ is assumed to be the same as the core; the separatrix density $\left(n_{e, \text { sep }}\right)$ for a H-mode is assumed equal to $n_{e} / 3$ (Kukushkin et al. 2001); and the separatrix temperature $\left(T_{e, \text { sep }}\right)$ is calculated iteratively assuming classical Spitzer-Harm conduction (Stangeby 2000). Despite the significant uncertainty associated with the scalings and the assumptions needed, it is reassuring that they all predict the heat flux width in SPARC to within a factor of 2.5 of each other, which increases our confidence in the extrapolation. However, to be conservative, the narrowest heat flux width of $0.18 \mathrm{~mm}$ is assumed for the SPARC design. The two-dimensional (2-D) modelling studies discussed in $\S 4$ adjust cross-field transport coefficients so as to match this target value of $\lambda_{q}$.

\subsection{Divertor radiation fraction}

As mentioned in the introduction, access to dissipative divertor regimes in SPARC is difficult to predict. One method is to make use of the simple 1-D models developed by Reinke (2017) and Goldston, Reinke \& Schwartz (2017) to examine the scaling for the impurity fraction needed to achieve detached divertor conditions with tokamak operating parameters (e.g. magnetic field, size). Recent experiment results from JET and ASDEX Upgrade have found that the nitrogen fractions needed to maintain detached 
divertor conditions in a power scan roughly agree with these scaling law predictions (Henderson et al. 2019), although the absolute magnitudes are still uncertain. Reinke (2017) estimates that to reach divertor target ion temperatures of $5 \mathrm{eV}$, a proxy for divertor detachment, a nitrogen impurity fraction of $11.4 \%$ is needed in a $1.65 \mathrm{~m}$ major radius, 12.0 $\mathrm{T}$ device that is running at a Greenwald fraction of 0.8. Scaling to SPARC V2 with $B_{0}, R_{0}$ and $f_{\mathrm{GW}}$ takes this up to a $33.5 \%$ nitrogen impurity fraction and $11.5 \%$ for neon. Such high-impurity fractions in the divertor will likely result in significant core contamination and affect fusion performance. As the design is currently focused on the primary reference discharge and it is unclear whether such a high-impurity fraction will be tolerable for the core plasma, rather than aim for $\sim 100 \%$ radiation fraction leading to detached or near-detached conditions, a moderate $50 \%$ radiation fraction is assumed for baseline design scenario of SPARC V2. As 2-D SOL simulations become more mature, the specification for the radiation fraction will be revisited.

It is important to note that a $50 \%$ divertor radiation fraction is not necessarily conservative, however, there are some mitigating factors that can used during SPARC operations to ensure divertor target survivability while still meeting the primary mission of $Q>2$. The high-impurity fraction calculated for SPARC V2 based on the scaling by Reinke (2017) is largely due the low target $f_{\mathrm{GW}}$, and the assumed ratio of $n_{e, \text { sep }}$ to the pedestal density $\left(n_{e \text {,ped }}\right)$. The former is a result of an administrative fusion power limit whereas the latter is driven by a desire to maximize pedestal performance. The current primary operating point is chosen to maximize fusion gain while limiting the total fusion power (Creely et al. 2020), but there exists a relatively large operational window that can meet the primary mission of $\operatorname{SPARC}(Q>2)$ at higher $f_{\mathrm{GW}}$. Note, however, that the plasmas at higher $f_{\mathrm{GW}}$ would have to be run 'lean' with a higher deuterium-to-tritium ratio (or larger impurity dilution) in order to keep within the fusion power limits. Similarly, the assumed ratio of $n_{e \text {,sep }}$ to $n_{e \text {,ped }}$ can be increased, but at fixed $f_{\mathrm{GW}}$, would imply reducing the pedestal pressure, likely reducing $Q$ from the primary reference discharge. As the primary reference discharge projects to $Q=11$, some reduction in core plasma performance is tolerable while still allowing SPARC to achieve its primary mission target of $Q>2$.

\subsection{Divertor target geometry}

The divertor target geometry must be designed to fulfil a number of roles: the target plates have to be shaped to maximize the surface area swept out by the strike point sweep (figure 1a) while at the same time ensuring that sufficient divertor volume is allocated for the XPT configuration (figure $1 b$ ). The divertor outline as shown in figure 1 is an approximate outline designed to meet these requirements. The features shown in the outer divertor are a result of combining the swept heat flux mission and the XPT divertor mission. Only during XPT operation is the outer strike point intended to intersect with the vertical and horizontal faces at larger major radius. The sweeping, outside of XPT operation, will be controlled to ensure that the strike point is kept on the angled, high heat flux surfaces highlighted in pink. The development of this divertor outline is in progress as considerations have yet to be made for disruption forces, installation, assembly, and maintenance. Furthermore, it has yet to be assessed how much space is required for the secondary X-point of the XPT divertor, which will be determined from an assessment of the diagnostic needs as well as simulations of the XPT divertor. Therefore, the divertor geometry will continue to evolve as the design and analysis of the SPARC divertor progresses.

The current divertor geometry shown in figure 1 allows for the inner divertor strike point to be swept over a target surface poloidal arc length of $0.3 \mathrm{~m}$, corresponding to an area of $\sim 2.6 \mathrm{~m}^{2}$. During the same sweep process the outer divertor strike point sweeps 
out a poloidal arc length of $0.4 \mathrm{~m}$, corresponding to an area of $\sim 4.5 \mathrm{~m}^{2}$. In addition, the magnetic field line surface incident angle varies across the high heat flux surfaces as the strike point is swept, but stays below $1^{\circ}$, assuming a perfectly axisymmetric target. However, machining and installation tolerances along with the needed chamfering of tile edges will increase the incident field line angle. Owing to this, 'fish-scaling' of the divertor tiles may be necessary to protect adjacent tiles, as was done for ITER (Pitts et al. 2017), where each tile surface is tilted by $1^{\circ}$. Therefore, to be conservative, an incident field line angle of $2^{\circ}$ is assumed for the SPARC V2 divertor thermal analysis; this will be reassessed as a more detailed design is produced.

\subsection{Divertor target heat flux profile}

To estimate the divertor target heat flux profile, the SPARC V2 primary reference discharge magnetic equilibrium is used to calculate the flux expansion to the divertor target. The divertor target surface plasma heat flux profile is approximated using the Eich et al. (2011a) profile of an exponential function convoluted with a Gaussian function of width $S$, where the multi-machine $\mathrm{H}$-mode database suggests that $S \sim \lambda_{q} / 2$ (Eich et al. 2013). Owing to divertor and SOL radiation, the total integrated power due to incident plasma heat flux profiles is less than $P_{\mathrm{SOL}}$, reducing the peak heat fluxes. Experimentally, as the radiation fraction increases and divertor plasma temperatures decrease, a broadening of the plasma heat flux profile is also observed (Kallenbach et al. 2015), which should further reduce the peak; however, as the exact scaling is unclear, these additional effects are not included.

Owing to the enclosed divertor geometry, the majority of the divertor radiation will be deposited on the divertor target surfaces. The radiation heat flux profile across the divertor target is dependent on the source locations and is difficult to predict for SPARC at present. Instead, for simplicity, the divertor radiated power is simply added to the plasma heat flux profile as a constant uniform background heat flux term, similar to that used in the multi-machine H-mode database study (Eich et al. 2013), to obtain the total surface heat flux profile. While this uniform background is much smaller, it is being applied continuously compared with the transient sweeping of the high heat flux feat and still plays a role in assessing the divertor survivability. Thus, total integrated power from the plasma heat flux profiles and the uniform radiation term across the entire divertor target surface is taken to equal $P_{\mathrm{SOL}}$. Assuming a $50 \%$ radiation fraction, this gives the heat flux profiles shown in figure 2 with peak heat fluxes at the inner and outer divertor of 257 and $357 \mathrm{MW} \mathrm{m}^{-2}$ respectively.

\subsection{Divertor ELM loading}

Through a study of the SPARC pedestal included in a companion paper (Hughes et al. $2020)$, it is anticipated that Type 1 ELMs size $\left(\Delta W_{\text {ped }}\right)$ in SPARC V2 will be greater than 1 MJ per ELM (14-22\% of the pedestal stored energy). To translate that ELM energy into divertor power loading we utilize a multi-machine database by Eich et al. (2017) which provides an approximate model for the peak parallel ELM energy fluence $\left(\epsilon_{\|, \text {peak }}\right)$ on the divertor target that has a linear dependence on the pedestal pressure. In that study the surface heat flux to the divertor is integrated in time across an ELM pulse and projected parallel to the magnetic field using the steady state magnetic field line incident angle. The $2^{\circ}$ incident field line angle assumed in $\S 2.5$ will be used here to project $\epsilon_{\| \text {,peak }}$ into a target surface thermal load. It has been experimentally observed that the ELM power deposition at the divertor target comes in two phases (Loarte et al. 2007; Eich et al. 2011b): an initial fast deposition phase where a significant fraction of the ELM energy is deposited on a short timescale $\left(\tau_{\mathrm{ELM}}\right)$ leading to a rise in the divertor target surface temperatures; and a second 


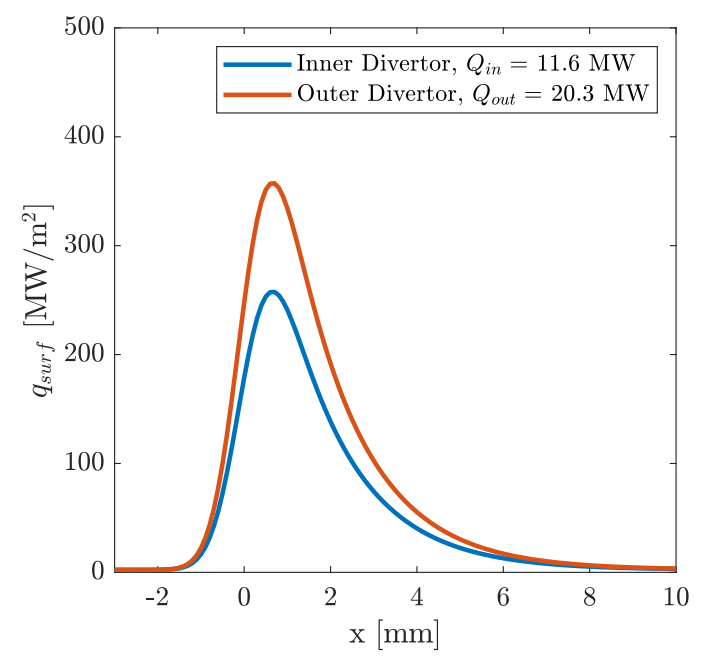

FIGURE 2. SPARC V2 divertor surface heat fluxes shown as a function of distance along the divertor target surface. The profiles assume a $50 \%$ divertor radiation fraction that is 'returned' to the profile as a uniform background heat flux term across the entire target surface resulting in the small non-zero values of $q_{\text {Surf }}$ at the ends of the profiles shown. The rest of the profile follows an Eich et al. (2011a) profile with $\lambda_{q}=0.18 \mathrm{~mm}$ and $S=\lambda_{q} / 2$. Note that the total integrated power over both divertors is $31.9 \mathrm{MW}$ and includes an additional $10 \%$ power owing to the conservative power-sharing assumptions.

slower phase where power continues to be deposited on the divertor targets at a lower level and the surface temperature begins to decrease. The initial fast phase is the most damaging on material surfaces and $\tau_{\mathrm{ELM}}$ has been experimentally observed to correspond to twice the pedestal transit time for ions in the SOL (Loarte et al. 2007). For SPARC V2, assuming a pedestal pressure of $325 \mathrm{kPa}$ and pedestal density of $n_{e \text {,ped }}=2.8 \times 10^{20} \mathrm{~m}^{-3}$, we find that $\tau_{\text {ELM }} \sim 0.120 \mathrm{~ms}$ and $\epsilon_{\| \text {,peak }} \approx 10.7-32 \mathrm{MJ} \mathrm{m}^{-2}$. Note the factor of three range in $\epsilon_{\| \text {,peak }}$, which roughly corresponds to the range in ELM loading observed in experiments between small and large ELMs. This is remarkably close to the energy fluence scaling projection for ITER at $\epsilon_{\| \text {,peak }} \approx 10-30 \mathrm{MJ} \mathrm{m}^{-2}$ (Eich et al. 2017).

A common figure of merit for transient thermal loading is the surface heat flux factor (Pintsuk et al. 2007). For a given material, the heat flux factor is a proxy for the resulting surface temperature rise based on an infinite semi-plane approximation. Approximately $15-40 \%$ of the ELM energy fluence is deposited during the first fast phase of an ELM (Loarte et al. 2007). Accounting for this and assuming an incident field line angle of $2^{\circ}, \epsilon_{\| \text {,peak }}$ and $\tau_{\text {ELM }}$ can be combined to calculate the surface heat flux factor for SPARC $\mathrm{V} 2: \mathrm{HHF}_{\mathrm{ELM}} \approx 5.1-41 \mathrm{MJ} \mathrm{m}^{-2} \mathrm{~s}^{-1 / 2}$. There is significant uncertainty in this prediction captured by the wide range in the expected transient thermal loading of the divertor targets, the upper end of which is close to the cited surface melt limit of tungsten $\sim 50 \mathrm{MJ} \mathrm{m}^{-2} \mathrm{~s}^{-1 / 2}$ (Pintsuk et al. 2007) and where cracks have been generated $\sim 30 \mathrm{MJ} \mathrm{m}^{-2} \mathrm{~s}^{-1 / 2}$ (Hirai et al. 2009). These ELM loads on a carbon divertor would lead to localized 'blooms' (Ulrickson, JET Team \& TFTR Team 1990). Current efforts within the team are focused on reducing the uncertainty to more tightly bound predictions.

ELM mitigation techniques are being assessed for SPARC, as is the potential to access intrinsically ELM suppressed regimes. Any ELM mitigation or suppression technique is likely to result in reduced operating pedestal. Nonetheless, core transport 


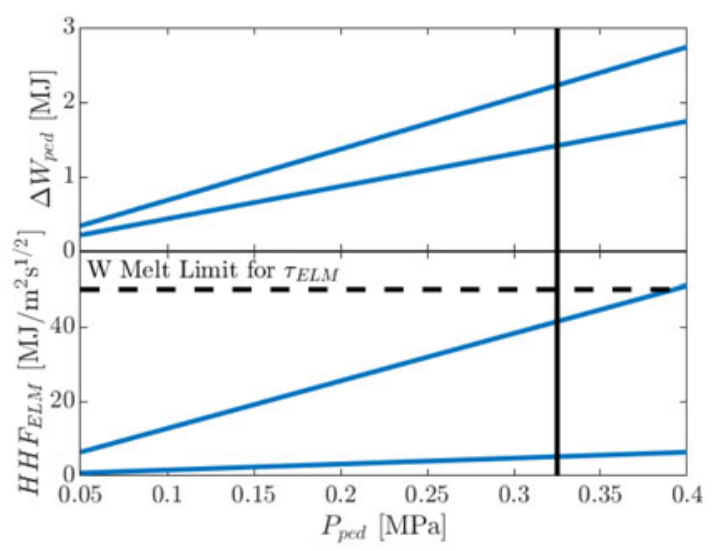

FIGURE 3. ELM size and the divertor peak surface heat flux predictions for SPARC V2 as a function of pedestal pressure. The two lines in each subplot bracket the range in the anticipated values. The critical surface melt limit for tungsten is shown given an expected $\tau_{\text {ELM }} \sim 0.12 \mathrm{~ms}$.

modelling indicates that even if the predicted pedestal pressure drops by half due to ELM suppression, core plasma performance will still meet the primary mission of SPARC with $Q>2$ (Hughes et al. 2020; Rodriguez-Fernandez et al. 2020). Figure 3, estimates the ELM size and $q_{\text {ELM,peak }}$ on the divertor as a function of pedestal pressure. A scheme for ELM mitigation in SPARC has yet to be developed, but this presents a potential pathway by which divertor survivability can be ensured while still meeting the primary mission of SPARC.

\subsection{Divertor disruption loading}

Thermal and structural loading during disruptions is a significant concern for SPARC and is the subject of a companion article (Sweeney et al. 2020) where the timescales and energy release are predicted. An unmitigated disruption can deposit up to $90 \%$ (Riccardo \& Loarte 2005) of the plasma stored energy (24.3 MJ) onto the divertor over the thermal quench timescale $(0.1 \mathrm{~ms})$. There is significant uncertainty as to the heat flux footprint during the thermal quench. Experimental measurements suggest that the footprint might grow by a factor of 3-10 (Riccardo \& Loarte 2005), or even up to a factor of 20 (Hender et al. 2007). Assuming that the steady-state power sharing between the inner and outer divertor as well as the divertor target surface incident field line angle, an unmitigated disruption results in a transient thermal loading to the divertor surface with a heat flux factor of approximately $5.3-36 \mathrm{GJ} \mathrm{m}^{-2} \mathrm{~s}^{-1 / 2}$. Note that this approximation only considers the stored thermal energy of the plasma and not the poloidal magnetic energy which could also contribute to surface heating of the PFCs (Lipschultz et al. 2011), but on a timescale that is one to two orders of magnitude longer. Even on the low end of this range, the heat flux pulse will result in flash melting for tungsten or a 'bloom' for carbon. Simulations are underway to determine whether such high transient thermal loads will result in bulk melting of the tungsten PFCs, which can lead to permanent deformation of the divertor target tile surfaces and negatively affect operations. Even if no bulk melting is expected, the flash melting of tungsten or the erosion of carbon PFCs would result in significant dust production, which can also negatively affect operations once a significant amount has accumulated: affecting core impurity content, as a fire hazard and for tritium retention. An emphasis is being placed on disruption mitigation in SPARC making use of 
the ITER 'disruption budget' approach to determine what would be acceptable operational protocols.

In addition to thermal loads, disruptions result in significant structural loads on PFCs. Eddy and halo currents that are driven by the disrupting plasma lead to significant mechanical stresses in the material and, more importantly, in structural mounting joints. These loads have been quantified by Sweeney et al. (2020) and efforts are underway to design in-vessel components that will be able to withstand these forces.

Both the steady-state heat fluxes (figure 2) as well as the transient loading from ELMs and disruptions can severely stress PFCs. The design of the SPARC divertor to safely accept the steady-state heat fluxes with strike point sweeping is discussed in the next section along with the plan of incorporating transient loads into the design process.

\section{Mitigation of steady-state heat load through strike point sweeping}

To mitigate the high steady-state surface heat fluxes shown in figure 2 , the baseline design scenario in SPARC is to sweep the strike point for the duration of the $10 \mathrm{~s}$ flattop. Owing to the relatively short pulse length in SPARC and the strike point sweeping, the divertor targets can be designed to operate without active cooling during the pulse. As such, they can withstand much higher heat fluxes than a long-pulse, actively cooled divertor because steady-state thermal equilibrium is never reached and the design is not limited by the coolant temperature and flowrates. Instead, the maximum heat flux is limited by how quickly and how often the strike point is moving past a point along the surface relative to the material's thermo-mechanical properties. With each pass of the strike point, the surface temperature of the PFC spikes locally, but cools dominantly via conduction into the bulk material. Repeated passes cause the surface temperature of the PFC to rachet up slightly, but the increase is small in comparison with the initial temperature rise when the strike point passes a given location. To examine this behaviour, and understand its limitations, a simple 1-D heat equation solver was built to simulate a $2 \mathrm{~cm}$ thick PFC with an insulated boundary condition at one edge (vessel or mounting plate) and the input surface heat flux as a function of time on the other edge. The 1-D heat equation was solved numerically using MATLAB's partial differential equation package. The initial temperature was set to $700 \mathrm{~K}$ for all the scans to mimic the average temperature rise of the divertor target surface close to the end of the $10 \mathrm{~s}$ flattop. The temperature dependent thermal properties of pyrolytic graphite were used (Incropera et al. 2007) in the carbon-based PFC analysis, to take advantage of its anisotropic thermal conductivity properties, the high in-plane $\kappa_{\|}$was used. The same qualitative results of these scans were observed when the temperature-dependent properties of tungsten were used and are, thus, not shown. The temperature-dependent material properties are shown in figure 4 for completeness. Blackbody radiation was not included, which can aid in suppressing the peak temperature achieved per sweep.

To start, the sensitivity of the strike point sweep to the assumptions discussed in $\S 2$ are assessed. First, the sensitivity of the peak temperature rise due to a passing strike point is examined as a function of the surface heat flux profile shape $\left(\lambda_{q}, S\right)$ and total power. The divertor heat flux assumptions used are the same as discussed in $\S 2$ but the radiation fraction is set to zero. Figure 5(a) shows two heat flux profiles at the two extremes of the $\lambda_{q}$ scaling predictions. Figure 5(b) shows the resulting peak change in surface temperature $(\Delta T)$ resulting from the heat flux profile being scanned past a point at $0.5 \mathrm{~m} \mathrm{~s}^{-1}$. As may be expected, the peak surface $\Delta T$ decreases with increasing values of $\lambda_{q}$. Increasing $\lambda_{q}$ from 0.18 to $0.4 \mathrm{~mm}$ (an increase by a factor of 2.2), results in the peak surface $\Delta T$ decreasing from 991 to $629 \mathrm{~K}$ (a decrease of $37 \%$ ). Figure 6 shows a similar profile shape scan but now as a function of $S / \lambda_{q}$, assuming $\lambda_{q}=0.18 \mathrm{~mm}$. Note that in figure $6(b)$, a 


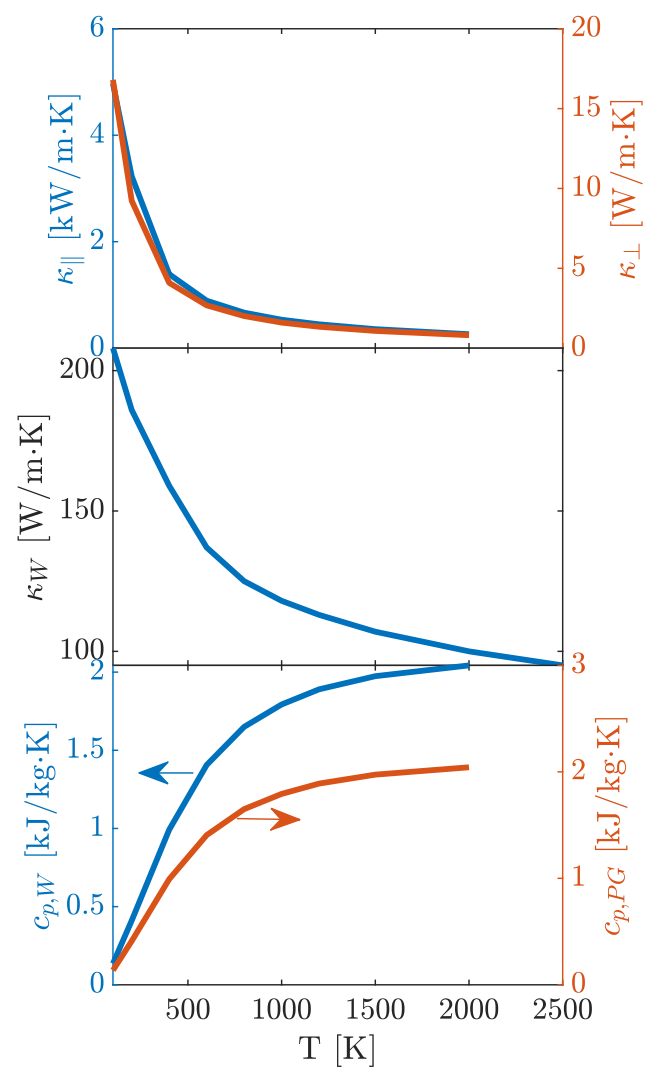

FIGURE 4. Temperature-dependent thermal properties of pyrolytic graphite and tungsten (Incropera et al. 2007). Top: the in-plane $\left(\kappa_{\|}\right)$and cross-plane $\left(\kappa_{\perp}\right)$ thermal conductivity of pyrolytic graphite note the different y-axis units. Middle: the thermal conductivity of tungsten. Bottom: the specific heat capacity of tungsten (blue) and pyrolytic graphite (orange).

second case with a different the total power to the outer divertor $\left(P_{\text {odiv }}\right)$ is given. As $S$ decreases, the peak surface heat flux increases but the peak surface $\Delta T$ starts to plateau. Conservation of energy would suggest that the saturation $\Delta T$ is linear with total power, which is generally consistent with the simulations, though there is some discrepancy because of the temperature-dependent thermal properties used. Figures 5 and 6 suggest that there is a clear benefit if the $\lambda_{q}$ is found to be larger than the most conservative empirical predictions, although the effect is less than linear. If $S$ turns out to be narrower due to suppressed cross-field transport in the divertor leg, the saturation in $\Delta T$ indicates that it is unlikely to cause a significant increase in divertor target surface temperatures.

We have also varied strike point sweep velocity and the fraction of $P_{\mathrm{SOL}}$ that is radiated in the divertor legs between the X-point and the target $\left(f_{\text {rad }}\right)$; see figure 7. As discussed in $\S 2.5$, the radiated power is applied back on the target surface as a uniform heat flux so as to conserve the same total power across the scan in $f_{\text {rad }}$. However, as the same profile is being swept at different velocities, the total 'exposure' time is a function of the strike point sweep velocity: the slower strike point sweep requires more time to pass a point and vice versa. As a result, for the $f_{\text {rad }}=1$ case, a flat uniform heat flux profile, $\Delta T$ is not constant with respect to sweep velocity. The power is constant but the total integrated energy being simulated decreases as the strike point velocity increases. In general, sweeping faster with 
(a)

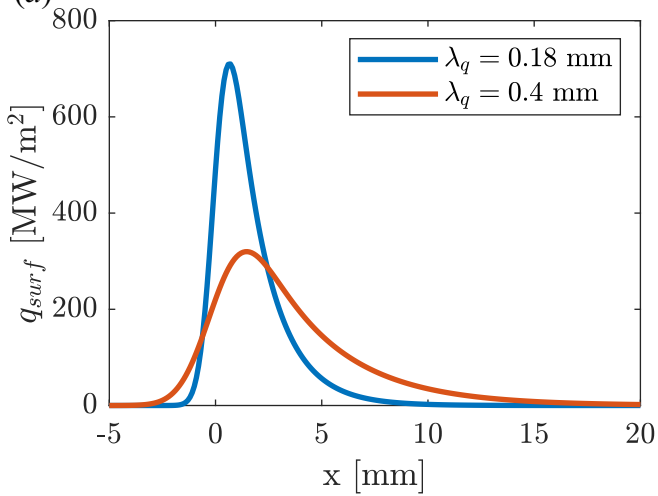

(b)

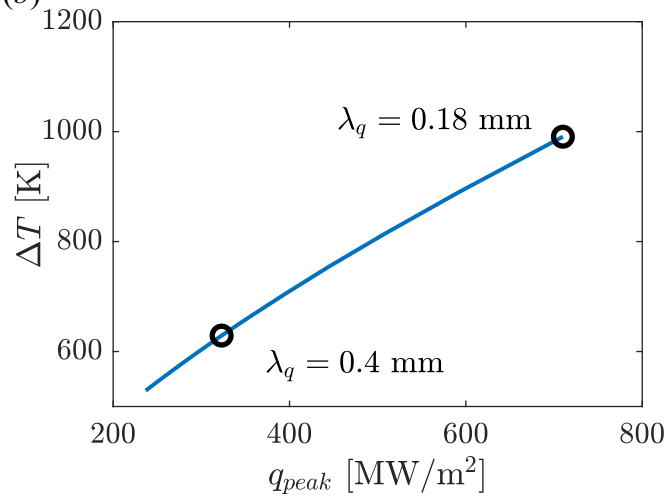

FIGURE 5. (a) The surface heat flux profile for two values of $\lambda_{q}$ as a function of the distance along the target surface. The profiles are consistent with the assumptions made in $\S 2$ with the exception of the radiated power fraction being set to zero. (b) The peak temperature of the passing strike point as a function of $\lambda_{q}$.
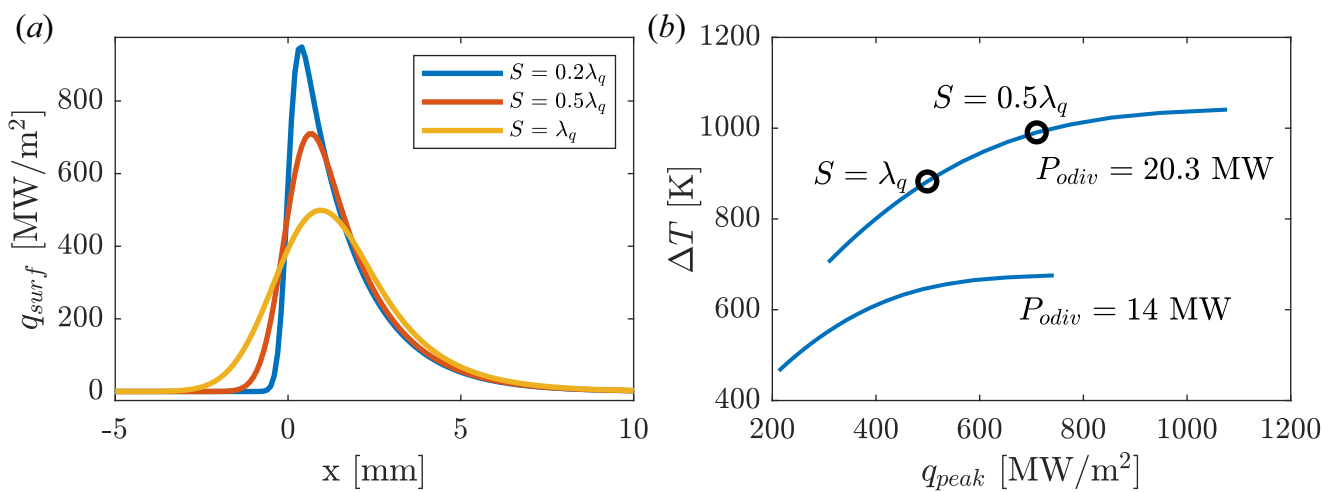

Figure 6. (a) The surface heat flux profile for $S=[0.2,0.5,1] \times \lambda_{q}\left(\lambda_{q}=0.18 \mathrm{~mm}\right)$ as a function of the distance along the target surface. The profiles are consistent with the assumptions made in this report with the exception of the radiated power fraction being set to zero. $(b)$ The peak temperature due to the passing strike point for two values of total power. Here $\Delta T$ plateaus as the peak surface heat flux increases.

a higher radiation fraction reduces the peak temperature on the divertor target. As the sweep velocities increase, the $\Delta T$ begins to asymptote indicating that there are diminishing returns as expected from consideration of the thermal time constants. The current design strike point sweep frequency is $\sim 0.8 \mathrm{~Hz}$, corresponding to a strike point sweep velocity of $0.5 \mathrm{~m} \mathrm{~s}^{-1}$ on the inner divertor and $0.7 \mathrm{~m} \mathrm{~s}^{-1}$ on the outer divertor. Although it would be good to be able to sweep the strike points faster, the power needed for the poloidal field coils for a strike point sweep frequency of $0.8 \mathrm{~Hz}$ exceed their power needs of the initial current ramp up, which are driving the power supply requirements for SPARC. Means to improve the sweep velocity by way of modifying the poloidal field coil designs and their power supplies are being assessed.

To obtain a quantitative prediction of the divertor target temperature response to the strike point sweep, a 2-D thermal model of the divertor target plate is simulated in COMSOL. A slab geometry was used, which will be updated when a more realistic 


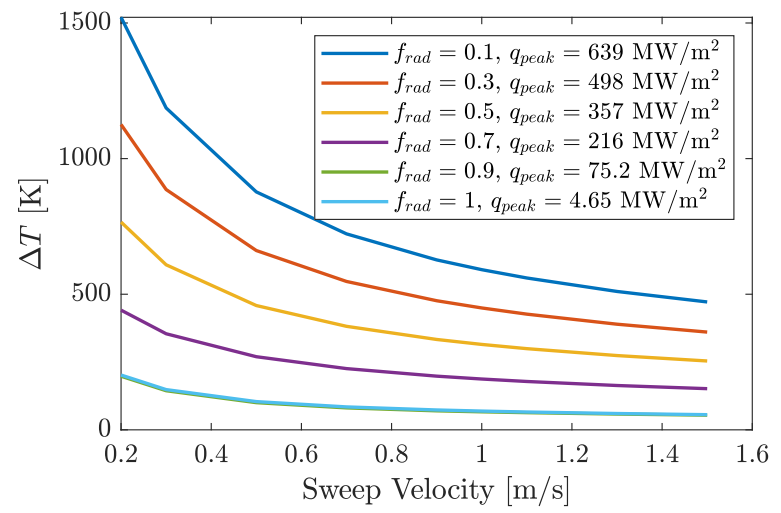

FIGURE 7. Scan of the peak change in temperature as a function of sweep velocity and radiation fraction. At the assumed $50 \%$ radiation fraction the peak surface $\Delta T$ starts to asymptote past $0.8 \mathrm{~m} \mathrm{~s}^{-1}$.
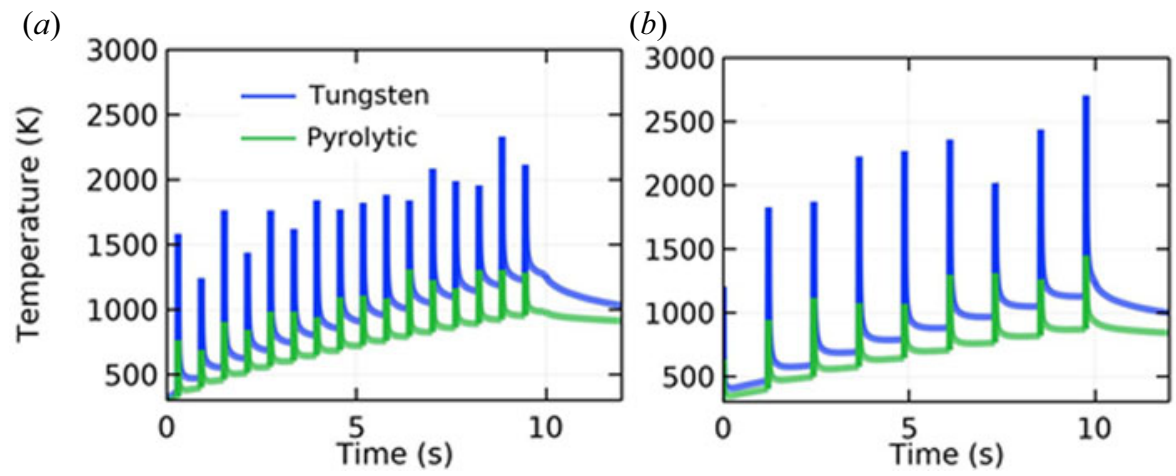

FIGURE 8. Simplified COMSOL 2-D thermal model of the outer divertor strike point sweep over a $10 \mathrm{~s}$ flattop and the subsequent cool-off for both a tungsten and pyrolytic graphite PFC material. The surface temperature of the PFC is plotted as a function of time at $(a)$ the middle of the sweep and $(b)$ near the turnaround point of the sweep. Note that the variation in $\Delta T$ for each peak is due to the coarseness of the computational grid.

divertor target model is available. The slab is $2 \mathrm{~cm}$ thick and $0.43 \mathrm{~m}$ long in the poloidal plane to simulate the outer divertor high heat flux target surface extent and the strike point is swept across at $0.7 \mathrm{~m} \mathrm{~s}^{-1}$. The temperature-dependent thermal properties shown in figure 4 of both pyrolytic graphite and tungsten (Incropera et al. 2007) were used, with the simulation initial temperature set to $298 \mathrm{~K}$. Note that the high in-plane thermal conductivity was only applied into the material, in the direction normal to the surface where the heat flux is applied. The lower cross-plane thermal conductivity is used in the other direction of the slab. This is a conservative approach because for the tile design, the planes of the material are aligned with the poloidal plane of the tokamak (the 2-D plane being simulated) with the 'poor' cross-plane direction of the material oriented in the toroidal direction. Figure 8 shows the simulation results of sweeping the outer divertor strike point for the $10 \mathrm{~s}$ duration of the flattop. We can see that given the current surface heat flux assumptions (figure 2) on the outer divertor target, the surface of a tungsten PFC would quickly cross the recrystallization temperature $(\sim 1700 \mathrm{~K})$ after a few passes. Thermal cycling of the material across the recrystallization temperature is likely to result 
in embrittlement and the formation of micro-cracks similar to that observed in laboratory ELM testing. Significant testing of tungsten PFCs will be performed to ensure that they can survive the design lifetime of the device. In contrast, the surface temperature excursions of the pyrolytic graphite simulation have some margin up to the carbon bloom temperature of 1900-2100 K (Ulrickson et al. 1990). Current efforts are focused on including the disruption structural loads in the PFC design and the ELM heat loads on top of the strike point sweep.

\section{Modelling of the SPARC divertor and access to dissipative divertor regimes}

Even though the baseline design scenario for operations is to take a conservative approach and assume moderate radiation fractions with an attached strike point, it is also of interest to understand what can be done to access dissipative divertor regimes. Standard boundary plasma-neutral simulation tools have been developed by the fusion boundary community and have been applied to simulate SPARC in UEDGE (Rognlien et al. 1992) and SOLPS-ITER (Weisen et al. 2015; Bonnin et al. 2016). The use of both codes will allow for cross-code comparisons to increase the confidence in their results and projections for SPARC. The SPARC operating parameters are significantly beyond the limits of validation of these simulation tools and at present, there are difficulties in verifying solutions and assessing convergence for such high-density and short-gradient scale-length plasmas. The modelling studies, although ongoing, are presented herein, along with a description of the proposed future work to assist in refining the baseline design guidelines for the SPARC divertor, and are designed to play to the strengths of the different packages. Furthermore, the Lengyel model as used by Reinke (2017) is applied to the SPARC V2 primary reference discharge to assess the impurity fraction needed to access moderate to high SOL radiation fractions and highlight the concern that the attainment and control of a dissipative divertor regime may be challenging at high power density and relatively low upstream plasma densities currently desired for optimizing the core plasma performance.

\subsection{Preliminary UEDGE simulations with $1 \%$ carbon}

In this section, we present results from UEDGE (Rognlien et al. 1992) modelling of the SPARC V2 double-null equilibrium in an up-down symmetric simulation. UEDGE solves the Braginskii fluid equations for the plasma with specified anomalous cross-field transport coefficients. A fluid model is utilized for the neutral transport that is appropriate for a short neutral mean free path regime as is anticipated in the SPARC divertor. For impurity radiation, the fixed-fraction impurity model is used and is tied to a fraction of the ion/electron density. Impurity radiation rates are obtained from the ADPAK code (Hulse 1983). The equations are discretized by a finite volume method on a 2-D spatial grid, and solved by fully implicit methods, finding exact steady states to machine precision.

For setting up the magnetic geometry, UEDGE uses the equilibrium of the SPARC primary reference discharge and creates a grid conformal to the magnetic flux surfaces in the region of $\psi_{N} \approx 0.98-1.02$, where $\psi_{N}$ is poloidal magnetic flux normalized to the value at the separatrix. The simulations are performed in the lower half-domain, below the midplane, assuming a balanced double-null configuration, with corresponding upstream profile and power sharing assumptions. For the simulation results shown here, the target plate is chosen to be normal to the flux surfaces. Boundary conditions are specified to match SPARC upstream operation parameters and to determine as accurately as possible the variables of interest at the outer boundary, approaching the limiter and walls. At the core interface, the boundary conditions use fixed plasma density, fixed ion and electron power and zero radial derivative of the parallel ion velocity. In the simulations, the total 

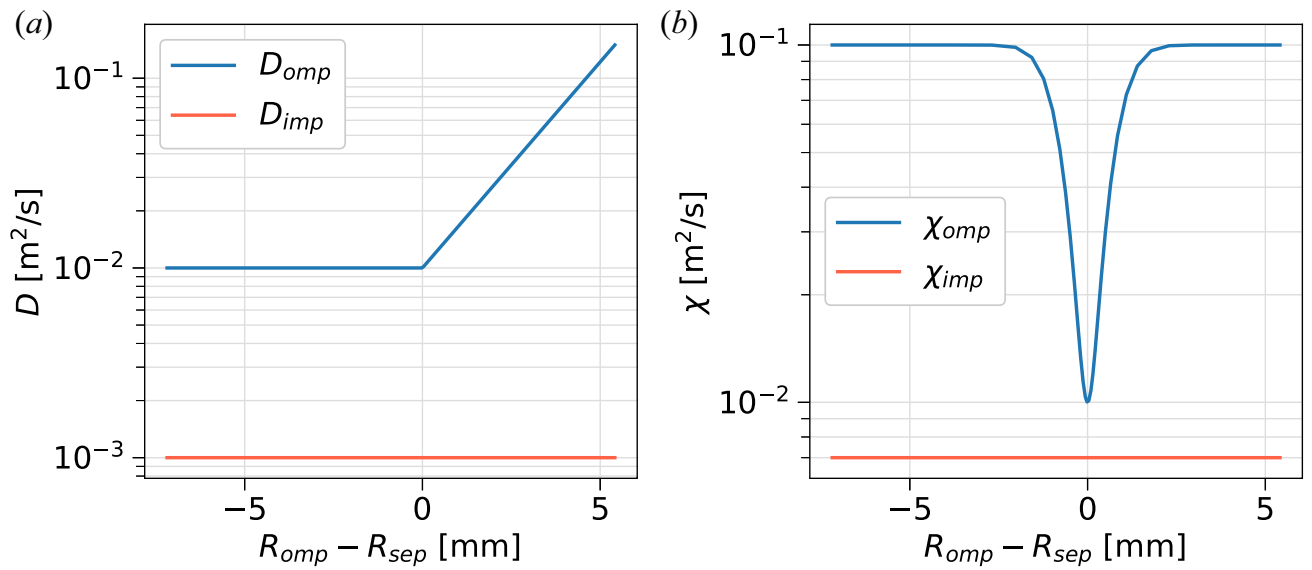

FIgURE 9. Particle $(D)$ and energy $(\chi)$ cross-field diffusion transport coefficients profiles at the inner (imp) and outer midplanes (omp).

power into the edge domain is generally set to $20 \mathrm{MW}$, although this will be pushed as high as $29 \mathrm{MW}$ to investigate SPARC operation at full power. The common flux region (edge of grid towards PFC surfaces) boundary conditions are extrapolated ion/electron density, ion temperature and electron temperature. The private flux region has boundary conditions for density and temperature specifying a gradient scale length of $1 \mathrm{~cm}$ (value divided by its derivative in the radial direction). Assuming that material surfaces reach saturation, the neutral recycling coefficient is set to 1 for all material wall boundaries. This means that each ion crossing the boundary reappears locally as a neutral, and none are injected into the system or pumped. The standard sheath boundary conditions are used at the divertor targets.

The cross-field diffusive transport coefficients relate density and energy fluxes to their gradients, i.e. $\Gamma_{n, \perp}=-D \nabla n$. The radial transport coefficients are user-specified to match predicted midplane plasma profiles, heat flux profiles on the target plates and inner/outer divertor power sharing, from empirical scalings and experimental data. The simulation domain was split into three regions: from the midplane to the X-point of the high field side (imp); from the midplane to the X-point on the low field side (omp); and the two divertor leg regions. The transport profiles shown in figure 9 were constant along flux surfaces in their respective domain. Across the entire simulation domain, the radial ion momentum diffusion coefficient is set to $0.5 \mathrm{~m}^{2} \mathrm{~s}^{-1}$. The radial energy diffusion coefficient $(\chi)$ for both ions and electrons is set to the values shown in figure 9, with low transport on the inboard side and enhanced transport with a barrier to simulation the pedestal on the outboard side. A flat $\chi$ of $0.1 \mathrm{~m}^{2} \mathrm{~s}^{-1}$ was used across the divertor legs consistent with outboard side value, but without the transport barrier. The depth of the energy transport barrier is used to control the exponential decay length of the parallel heat flux profile at the entrance to the outer divertor. Decreasing $\chi$ on the inboard side results in decreased power to the inner divertor target, and this was set such that the divertor power sharing was $1: 4$ near the X-point at full power with no impurities consistent with experimental observations of double-null power sharing. The radial density diffusion coefficient, $D$, is set to the values shown in figure 9. $D$, along with the core density, is set to obtain the desired upstream density profile. The inboard and outboard $D$ profiles shown in figure 9 were constant along flux surfaces all the way from the midplane to the divertor target. The $D$ profile which rises in the radially outward direction on the outboard side is a stand-in 

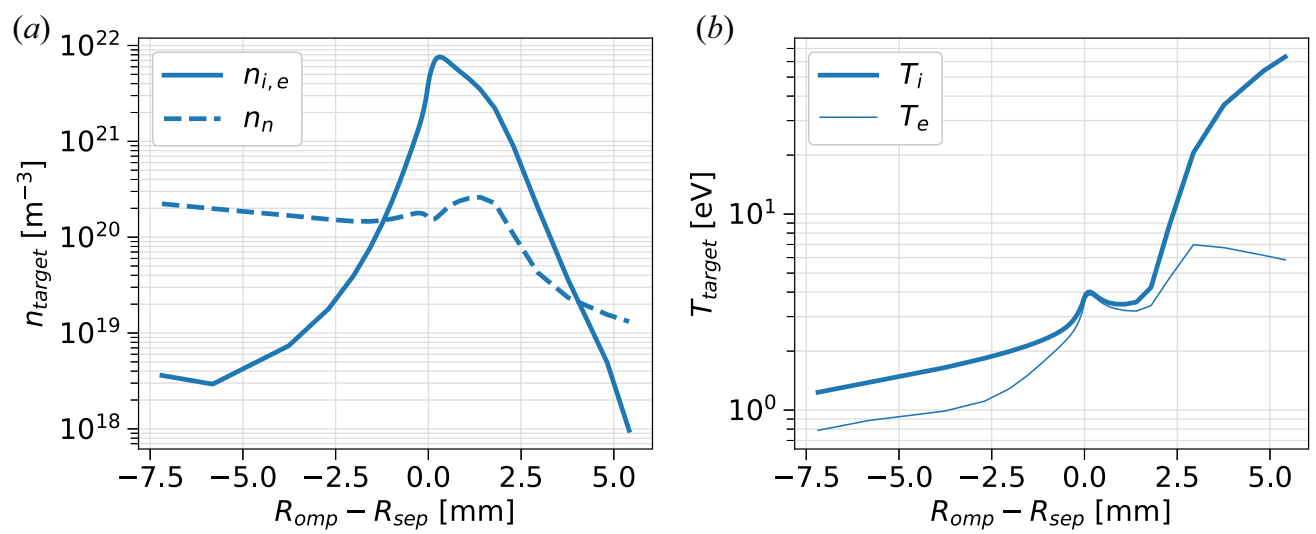

FIGURE 10. Density and temperature profiles at the outer divertor target plate mapped along flux surfaces to the outer midplane.

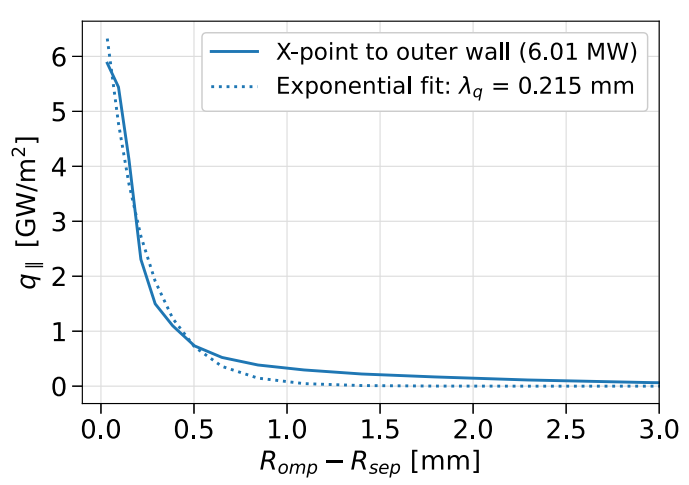

FIGURE 11. Convected and conducted power including electron, ion and neutral energy flux parallel to the magnetic field at the outboard side X-point, mapped along flux surfaces to the outer midplane. The total power crossing into the outer divertor is shown in parentheses in the legend. The dashed line shows a single exponential fit to the data from which the heat flux width is obtained.

for convective transport (Umansky et al. 1998). UEDGE runs including the convective transport terms are being developed. Some of the presented simulation parameters are also adjusted in individual cells to ensure converging solutions; the solver sometimes has difficulty converging owing to cells with very low density, especially near the end points of the divertor targets. Experimental data informing the value of the diffusion coefficients everywhere in the plasma are scarce, and no data exist on long-legged divertor transport, so the values used here are mostly ad hoc and tuned to match experimental profile predictions where available for fully attached divertor conditions with no impurities.

A case with $1 \%$ carbon fraction, $P_{\mathrm{SOL}}=20 \mathrm{MW}$, and a separatrix density of $1 \times$ $10^{20} \mathrm{~m}^{-3}$ is shown in figures $10-12$. In figure 10 , the density and temperature at the outer divertor target are mapped to the outer midplane. Target plate plasma temperatures are low while densities are high, indicating high recycling conditions. The inner target profiles are not shown, but and the target plasma temperature $(\sim 3.5 \mathrm{eV})$ is reduced significantly from midplane values and the target surface heat flux has also dropped to manageable levels of $<1 \mathrm{MW} \mathrm{m}^{-2}$. Figure 11 shows the convected and conducted power from ions, electrons and neutrals entering the outer divertor, in the direction parallel to the magnetic field near the 


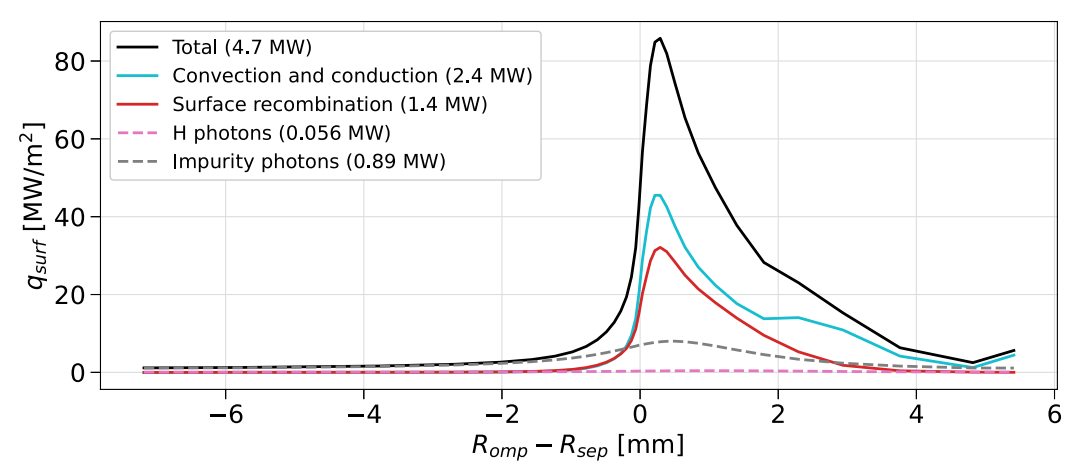

FIgURE 12. Total power flux to outer divertor target and its components mapped along flux surfaces to the outer midplane. The total power of each component is shown in parentheses in the legend.

X-point. A single exponential fit to the data indicates a heat flux width of $0.215 \mathrm{~mm}$, close to the empirical prediction of $0.18 \mathrm{~mm}$ by Eich et al. (2013). Finally, a breakdown of the power to the outer divertor target is shown in figure 12. Convection, conduction and surface recombination are the dominant contributors; radiation has relatively little effect. This picture is different for the inner divertor target, where heat flux to the surface is dominated by radiation. The broadening of the heat flux profile relative to the $\mathrm{X}$-point is being investigated. There are likely some effects owing to neutrals and side-wall interactions in the long-leg divertor geometry (Umansky et al. 2019). These power loss mechanisms might explain the low target temperatures despite the relatively low impurity fraction.

These results suggest that a combination of the long tightly baffled divertor leg geometry used in the simulations and a low level of impurities may be sufficient to significantly reduce the peak heat fluxes to the divertor target as compared with the profiles shown in figure 2. However, care should be taken in the interpretation of quantitative results from a single UEDGE simulation owing to the significant uncertainty in the assumed cross-field transport coefficients and other inputs. Sensitivity scans are planned to increase the overall confidence. Furthermore, UEDGE simulations are being developed to provide a wide 2-D scan in both power and impurity fraction with carbon and neon. This will help provide a map of the parameter space at which detachment is possible and the overall qualitative trends that can be used to inform divertor design decisions. Additional simulations are also planned for lower single-null discharges. These full domain simulations will allow for drift terms to be included and will provide high-fidelity predictions of SOL and divertor behaviour in SPARC.

\subsection{Preliminary SOLPS simulation results}

The SOLPS-ITER code (Bonnin et al. 2016) is applied to SPARC to study particle balance and impurity seeding properties of SPARC. Specifically, the simulations will be used to inform requirements on the fuel ion puffing location and flow rate, estimate the neutral pressure in the sub-divertor as needed to design pumping systems and estimate impurity flow rate requirements and optimal puffing location to reduce the divertor heat flux via dissipation. Simulations are performed for SPARC V2, but in an unbalanced double null topology biased towards the lower divertor. The distance between the two $\mathrm{X}$-points when mapped along flux surfaces to the outer midplane is $2 \mathrm{~mm}$, which is large compared with the baseline design SOL heat flux width discussed in $\S 2.2$. Figure 13 shows the SOLPS-ITER grid, with the coloured regions used for plasma and neutral 


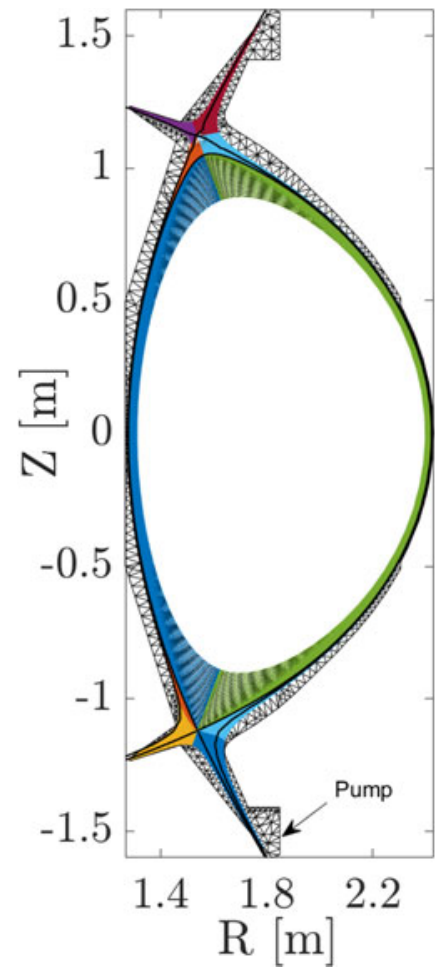

FIGURE 13. SOLPS-ITER grid used for SPARC simulations. Coloured regions indicate mesh for plasma transport, whereas the coloured mesh and black triangles are available for kinetic neutral transport.

transport, and the black triangulated region treated as vacuum for neutral transport only. The initial simulations shown here are unseeded. The code inputs are core boundary conditions of $29 \mathrm{MW}$ of power into the SOL, equally split between electrons and ions, and a density of $1.5 \times 10^{20} \mathrm{~m}^{-3}$, which corresponds to a separatrix density at the outboard midplane of $1.0 \times 10^{20} \mathrm{~m}^{-3}$. The indicated surface is used to pump neutral particles, with a recycling coefficient of 0.9 . Cross-field transport coefficients of $\chi_{\perp}=0.01 \mathrm{~m}^{2} \mathrm{~s}^{-1}$ and $D_{\perp}=0.1 \mathrm{~m}^{2} \mathrm{~s}^{-1}$ are chosen to reproduce the extrapolated heat flux width (figure 14), an exponential fit near the separatrix gives a decay length of $0.14 \mathrm{~mm}$.

Figure 15 shows profiles of the electron density and temperature, as well as the particle and heat fluxes carried by the plasma at the lower outer divertor. Without seeding the divertor is strongly attached, with high electron temperature $\left(T_{e}^{\text {peak }} \approx 300 \mathrm{eV}\right)$ and modest density $\left(n_{e}^{\text {peak }} \approx 1.7 \times 10^{21} \mathrm{~m}^{-3}\right)$. The peak heat flux density $q_{\text {surf }}^{\text {peak }}$ is $\approx 180 \mathrm{MW} \mathrm{m}^{-2}$, with $\sim 22 \mathrm{MW}$ of power deposited at the outer target. Compared with figure 2 , the difference in peak surface heat flux is largely due to the lower incident angle of the magnetic field at the target used in the SOLPS simulation $\left(\sim 0.8^{\circ}\right.$ at the OSP, $2^{\circ}$ in figure 2$)$. Accounting for this difference, there is reasonably good agreement between the SOLPS modelling and simple estimates with the Eich model, which increases confidence in the simulation output. The peak particle flux density $\Gamma_{\perp}^{\text {peak }}$ is $\sim 10^{24} \mathrm{~m}^{-2} \mathrm{~s}^{-1}$, occurs further into the SOL as compared with $q_{\text {surf }}^{\text {peak }}$, and is similar in magnitude to typical values for partially detached conditions in ITER (Pitts et al. 2019). The values are similar despite the different transport regimes, because of the much higher divertor $T_{e}$ in SPARC. 


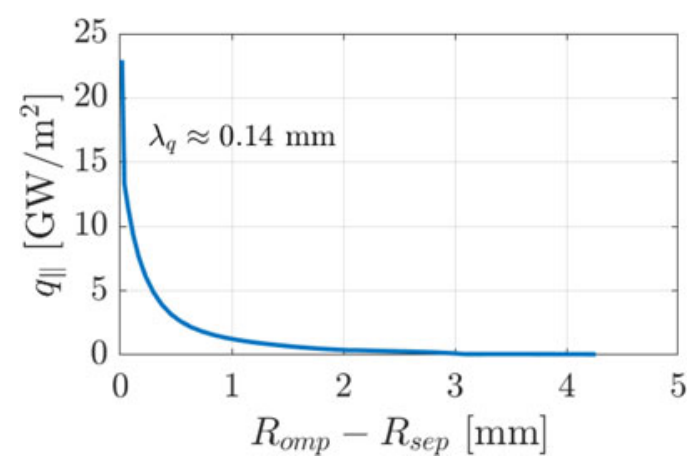

FIGURE 14. Parallel heat flux density at the outboard side X-point, mapped along flux surfaces to the outer midplane. Note that the parallel heat flux shown is higher than in figure 11 of the previous UEDGE simulations that used a lower $P_{\mathrm{SOL}}$ and assumed perfectly balanced double-null power sharing.

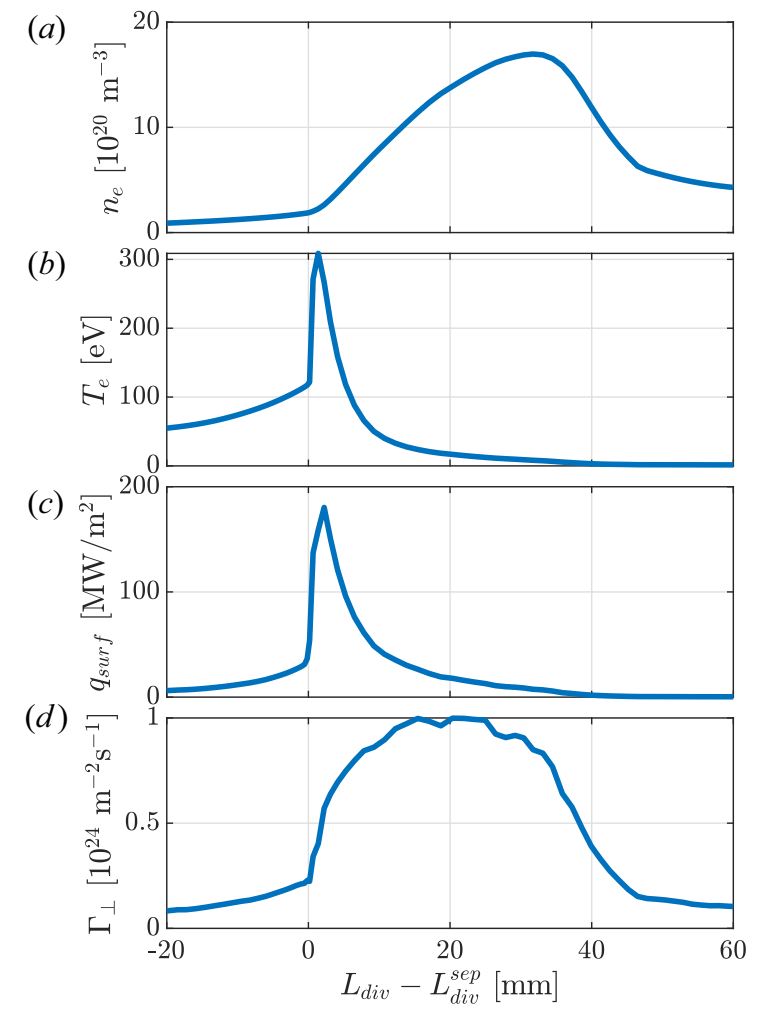

FIGURE 15. Radial profiles as a function of distance along the outer divertor target of $(a)$ electron density, $(b)$ electron temperature, $(c)$ deposited heat flux carried by the plasma and (d) deposited ion particle flux.

\subsection{Lengyel model estimates of impurity fraction}

As a parallel scoping of the operating space of the divertor versus impurity fraction we have utilized the simple Lengyel model applied by Reinke (2017). The model derives the required impurity fraction that is consistent with a target temperature that both satisfies the desired reduction of $q_{\|}$due to radiation as well as the sheath heat flux boundary 
(a)

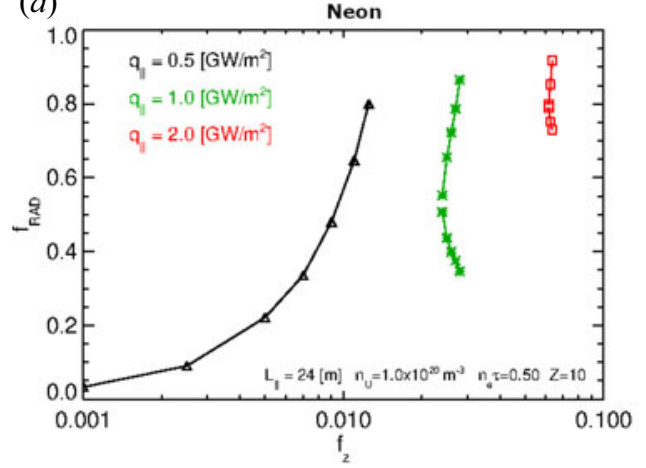

(b)

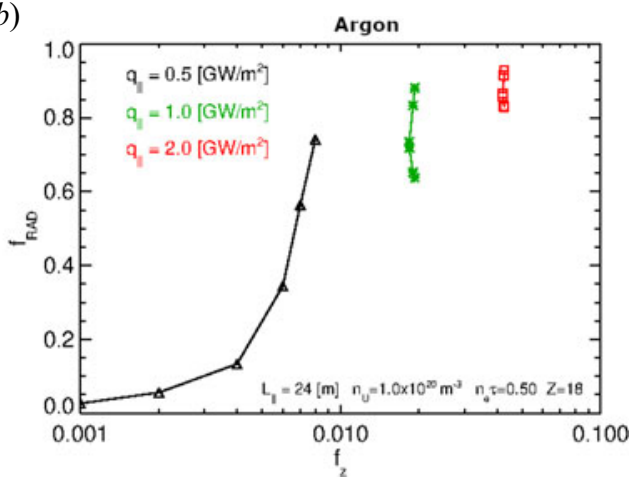

FIGURE 16. The radiation fraction $\left(f_{\text {rad }}\right)$ as a function of the impurity fraction $\left(f_{Z}\right)$ for $(a)$ neon and $(b)$ argon, calculated using the Lengyel model.

condition, assuming a sheath heat flux coefficient of $\gamma=7$. This is shown in figure 16 for connection length, $L_{\|}=24 \mathrm{~m}$, upstream density, $n_{u}=1.0 \times 10^{20} \mathrm{~m}^{-3}$, and using a non-coronal radiation model for neon and argon assuming a finite impurity residence time, $n_{e} \tau=0.5 \times 10^{20} \mathrm{~m}^{-3} \mathrm{~ms}$.

SPARC highlights an interesting behaviour found in its parameter space that is a result of its access to high parallel heat fluxes and low $f_{\mathrm{GW}}$ operational point. At low upstream $q_{\|} \sim 0.5 \mathrm{GW} \mathrm{m}^{-2}$ there is the expected behaviour that as the impurity fraction is increased, the SOL radiation fraction increases monotonically. An arbitrary SOL radiation fraction is possible, although the non-linearity suggests that control may be a challenge. At higher $q_{\|}$, the behaviour fundamentally shifts, and unless the impurity fraction reaches a higher level there is no solution, i.e. there is no target temperature where the radiation fraction reaches tens of percent and the sheath heat flux boundary condition is satisfied. The lack of a solution may be due in part to the Lengyel model analysis assuming a conduction-limited SOL which is not realized at high $q_{\|}$and low $n_{u}$ where a sheath-limited SOL is expected. This is interpreted as due to the high attached target temperature, the SOL can progressively fill with impurities, in this case neon or argon, without causing any substantive radiation until a tipping point is reached, the target temperature collapses and the radiation fraction jumps to a high level. This 'minimum' radiation fraction increases progressively as $q_{\|}$is increased as shown in figure 16. Furthermore, there exists a hot and cold branch where the sheath heat flux boundary condition can be satisfied, owing to the non-linear nature of the impurity cooling curves. Increasing the upstream density moves the transition to this bifurcated behaviour to higher $q_{\|}$, and it is also sensitive to the selection of impurity and assumption of its $n_{e} \tau$. The Lengyel model suggest that SPARC may not have arbitrary control over its divertor radiated power. When target plate temperatures start to decreases from the $\sim 300 \mathrm{eV}$ predictions of the SOLPS simulations (figure 15), divertor radiation fractions may already be significantly higher than the $50 \%$ assumed in $\$ 2$. This is in contrast to the results from UEDGE and efforts are underway to understand the discrepancies. Further analysis is required to understand the behaviour of the model and comparisons made to the other simulations to increase the confidence in this result.

\section{SPARC XPT divertor}

Divertor tokamaks rely on 'conventional' configurations known as the 'vertical target' (C-Mod, JET, AUG, ITER) and 'horizontal target' (DIII-D, JET) that have been in use 
for many years and are fairly well understood. In order for these 'conventional' divertors to be viable in a fusion pilot plant, efforts have grown to address whether large core radiation fractions (e.g. $95 \%$ ) can be used to reduce $P_{\mathrm{SOL}}$ in a reactor to levels that a conventional vertical target will accept safely, $<10 \mathrm{MW} \mathrm{m}^{-2}$ (Kukushkin et al. 2013; Wischmeier, The ASDEX Upgrade Team \& JET EFDA Contributors 2015; Bernert et al. 2017). 'Advanced' divertor configurations have been pushed forward as an alternative solution that may not require such high core radiation fractions, and experiments are currently being pursued or are planned on a number of different devices: TCV (Reimerdes et al. 2017), MAST-Upgrade (Katramados et al. 2011), ASDEX-Upgrade upper divertor (Lunt et al. 2017) and Italian-DTT (Ambrosino et al. 2019). The configurations that have been actively discussed within the boundary plasma community to-date include: (a) snowflake; (b) X-divertor; (c) Super-X (SXD); and XPT. Simulations have suggested that these advanced divertors have significant advantages over 'conventional' configurations in terms of their access to dissipative divertor regimes and their ability to provide a wide detachment power window (Umansky et al. 2017). Of the 'advanced' divertors, the XPT divertor geometry was found to have the widest detachment power window, which is advantageous from the perspective of power plant operations (Kuang et al. 2018). Therefore, to assess the performance of the XPT divertor at reactor-level power and plasma densities, it is being incorporated into the SPARC V2 design (figure $1 b$ ).

Owing to the high coil currents needed to pull the secondary X-point, the XPT divertor geometry is only accessible at somewhat reduced plasma current $\left(B_{0}=12.2 \mathrm{~T}, I_{p} \leq\right.$ 5.7 MA). Even then, preliminary assessment of the core performance using the POPCON analysis discussed in Creely et al. (2020) indicate that $Q \sim 1.9$ with $37 \mathrm{MW}$ of fusion power may be achievable. For the divertor this corresponds to $P_{\mathrm{SOL}}=22 \mathrm{MW}$ and a heat flux width of $\sim 0.25 \mathrm{~mm}$ (based on Eich et al. 2013), which maps to a parallel heat flux entering the divertor that is higher than in present-day devices. Some of the key issues that SPARC will attempt to address with the XPT divertor geometry include the sensitivity of power dissipation to the secondary null location in flux space relative to the primary separatrix, how sensitive the divertor detachment location and dissipation is to external controls and its response to transients. Although it has yet to be started, modelling of the XPT with UEDGE and SOLPS-ITER is planned to help optimize the design and determine the optimal feedback scheme.

\section{Discussion}

Table 3 summarizes how SPARC V2 compares against present-day devices, ITER and ARC for the normalized maximum conducted power entering the SOL. Note that the maximum conducted power used in the table is defined as the maximum available auxiliary power in present-day devices, whereas for SPARC V2, ITER and ARC, the projections for $P_{\text {SOL }}$ assuming no core radiation are used. Though a simplification, table 3 nonetheless provides an understanding of the extrapolation involved, and the significant uncertainty, in projecting experimental results from present-day devices to SPARC V2, or worst, to an ARC-class pilot plant. As a result of the large uncertainty, the conservative approach had to be taken when outlining the baseline design scenario for the SPARC V2 divertor or potentially risk SPARC failing to reach its primary mission of $Q>2$. Even without the added effect of transient loading from ELMs and disruptions, simulations of the strike point sweeping in $\S 3$ shows that the conservative baseline design scenario is pushing the limits of materials to withstand these heat fluxes. Using this same conservative approach, it will likely be impossible to design a divertor for an ARC-class device. This highlights the importance of intermediary devices such as SPARC V2 and ITER for reducing the uncertainty in developing design guidelines for a future fusion pilot plant. SPARC will be 


$\begin{array}{lcccccc}\text { Symbol } & \text { ASDEX-upgrade } & \text { JET } & \text { C-Mod } & \text { SPARC V2 } & \text { ITER } & \text { ARC } \\ B_{0}[\mathrm{~T}] & 3.9 & 3.7 & 8 & 12.2 & 5.6 & 9.2 \\ R_{0}[\mathrm{~m}] & 1.65 & 2.96 & 0.67 & 1.85 & 6.2 & 3.3 \\ P_{\mathrm{SOL}, \max }[\mathrm{MW}] & 30 & 40 & 6 & 41 & 150 & 144 \\ \frac{P_{\mathrm{SOL}, \max } B_{0}}{R_{0}}\left[\mathrm{MW} \cdot \mathrm{T} \mathrm{m}^{-1}\right] & 71 & 50 & 84 & 270 & 135 & 400\end{array}$

TABLE 3. A comparison of the normalized maximum SOL conducted power for ASDEX-Upgrade (Streibl et al. 2003), JET (Garzotti et al. 2019), Alcator C-Mod (Marmar \& Alcator C-Mod Group 2007), SPARC V2 (Creely et al. 2020), ITER (Casper et al. 2013) and ARC (Kuang et al. 2018). Note that the maximum conducted power ( $\left.P_{\mathrm{SOL} \text {, max }}\right)$ corresponds to the maximum available auxiliary power for the present-day devices whereas for SPARC V2, ITER and ARC it refers to the projections for $P_{\mathrm{SOL}}$ assuming no core radiation.

a valuable platform for providing crucial data for designing the divertor in a fusion pilot plant. How valuable, though, will depend on the available diagnostic set that is currently being evaluated.

The focus of diagnostics on SPARC will be to inform future divertor design efforts. Potential areas for investigation would include, but are not limited to: divertor target power sharing and the ability to maintain double null; the divertor target heat flux profile; and access to dissipative divertor regimes. Conservative assumptions had to be made in $\S 2$ with regards to these topics when developing the design guidelines for SPARC V2 owing to the uncertainty in the extrapolations. Operations on SPARC will help to inform how well perfect double-null power sharing has to be maintained to share power between the upper and lower divertors as well as reduce power to the inner divertors. If it has to be maintained to within $\sim \lambda_{q}$, then it could potentially have a major effect on power plant divertor designs. Understanding how power-sharing measurements may scale and affect next step device design, though, depends on the ability to measure $\lambda_{q}$ on SPARC. Combined, this will inform the magnitude of the heat exhaust challenge for a fusion pilot plant. Between the various scalings shown in $\S 2.2$, there is a factor of 2.5 range in the projections to SPARC V2. In addition, results from codes such as XGC predict a broadening of the heat flux width for ITER (Chang et al. 2017). A prediction of the $\lambda_{q}$ in SPARC by XGC has yet to be made. Although not as low as in ITER, the SPARC V2 reference H-mode has a $\rho^{*}=0.0027$ (Creely et al. 2020). It is possible that the increase in the turbulent electron effects proposed as the driving mechanism for the broadening in the predicted $\lambda_{q}$ of ITER may be observed in SPARC (Chang et al. 2018). Measurements of SPARC V2 $\lambda_{q}$ will certainly be an important data point for benchmarking empirical scalings and validating first principles computational models. Lastly, the inclusion of the XPT divertor geometry in SPARC is driven by the desire to assess its viability for an ARC-class device. Key issues of interest for this topic have already been mentioned in $\S 5$.

\section{Conclusions}

In conclusion, heat flux mitigation will be a key challenge for SPARC operations and an opportunity to extend our experimental knowledge into the fusion power regime, with the baseline divertor design scenario projecting to peak perpendicular surface heat fluxes on the inner and outer divertor target of $\sim 250$ and $\sim 350 \mathrm{MW} \mathrm{m}^{-2}$ respectively. Initial simplified calculations of the divertor target surface heat loads suggest that melt temperatures for tungsten and 'bloom' thresholds for carbon will not be reached if the 
strike point is swept at $0.8 \mathrm{~Hz}$ across the target surfaces for the duration of the $10 \mathrm{~s}$ flattop. Transient loading from ELMs and disruptions have yet to be incorporated into the design of the PFCs; however, estimates of the thermal and structural loads have been made with wide uncertainties remaining owing to the significant extrapolations made to conditions perceived for SPARC. As in ITER, these appear to challenge the ability of materials to withstand unmitigated transient fluxes without damage. Dissipative divertor operation may not be needed for achieving the SPARC primary mission of $Q>2$ but SPARC represents a unique opportunity to study divertor operations under reactor conditions. Simulation efforts are currently being made to develop predictions of SOL and divertor conditions to inform design decisions to ensure that dissipative divertor regimes are not precluded. Furthermore, SPARC is being designed to allow for XPT divertor operations. Even at the reduced plasma current needed to pull the secondary X-point into the divertor volume, with a $P_{\text {SOL }}=22 \mathrm{MW}$ and a heat flux width of $\sim 0.25 \mathrm{~mm}$, it will still present a significant heat exhaust challenge for the divertor and will be useful in assessing whether the XPT divertor geometry is a viable option for a future fusion pilot plant. Ideally measurements made on SPARC will be able to reduce significantly the uncertainty in projections of divertor performance and to increase the confidence in the design of a fusion pilot plant.

\section{Acknowledgements}

This work includes the contributions and funding of scientists from a number of organizations: SPARC itself was funded by Commonwealth Fusion Systems under RPP005; T.G. and S.B. were funded in part under INFUSE grants awarded to Commonwealth Fusion Systems; INFUSE is a DOE SC FES private-public partnership programme; M.L.R., J.D.L., and J.C. were funded by CFS through an ORNL Strategic Partnership Project \# NFE-19-07728; S.B. was also funded in part by the SPARC Fellowship Fund and the S. W. Ing (1953) Memorial Fund, a gift of Mimi and Frank Slaughter; and the work performed by M.U. at LLNL was supported by DoE Contract DE-AC52-07NA27344.

Editor William Dorland thanks the referees for their advice in evaluating this article.

\section{Declaration of interests}

The authors report no conflict of interest.

\section{REFERENCES}

Ambrosino, R., Castaldo, A., Ramogida, G., Villone, F., Albanese, R., Crisanti, F., MARTin, P. \& PizzUto, A. 2019 Magnetic configurations and electromagnetic analysis of the Italian DTT device. Fusion Engng Des. 146 (Part A), 1246-1253.

Andrew, P., Brennan, P. D., Coad, J. P., Ehrenberg, J., Gadeberg, M., Gibson, A., Hillis, D. L., How, J., Jarvis, O. N., Jensen, H., et al. 1999 Tritium retention and clean-up in JET. Fusion Engng Des. 47 (2-3), 233-245.

Bernert, M., Wischmeier, M., Huber, A., Reimold, F., Lipschultz, B., Lowry, C., Brezinsek, S., Dux, R., Eich, T., Kallenbach, A., et al. 2017 Power exhaust by SOL and pedestal radiation at ASDEX upgrade and JET. Nucl. Mater. Energy 12, 111-118.

Bonnin, X., Dekeyser, W., Pitts, R., Coster, D., Voskoboynikov, S. \& Wiesen, S. 2016 Presentation of the new SOLPS-ITER code package for tokamak plasma edge modelling. Plasma Fusion Res. 11, 1403102.

Brunner, D., Wolfe, S. M., LaBombard, B., Kuang, A. Q., Lipschultz, B., Reinke, M. L., Hubbard, A., Hughes, J., Mumgaard, R. T., Terry, J. L., et al. 2017 Surface heat flux feedback controlled impurity seeding experiments with Alcator C-Mod's high-Z vertical target plate 
divertor: performance, limitations and implications for fusion power reactors. Nucl. Fusion 57 (8), 086030.

Brunner, D., Kuang, A. Q., LaBombard, B. \& Terry, J. L. $2018 a$ The dependence of divertor power sharing on magnetic flux balance in near double-null configurations on Alcator C-Mod. Nucl. Fusion 58 (7), 076010.

Brunner, D., LaBombard, B., KuAng, A. Q. \& Terry, J. L. $2018 b$ High-resolution heat flux width measurements at reactor-level magnetic fields and observation of a unified width scaling across confinement regimes in the Alcator C-Mod tokamak. Nucl. Fusion 58 (9), 094002.

Casper, T., Gribov, Y., Kavin, A., Lukash, V., Khayrutdinov, R., Fujieda, H., Kessel, C. \& AgEnCIES, I. D. 2013 Development of the ITER baseline inductive scenario. Nucl. Fusion 54 (1), 013005.

Chang, C. S., Ku, S., Loarte, A., Parail, V., Koechl, F., Romanelli, M., Maingi, R., Ahn, J. W., Gray, T., Hughes, J., et al. 2017 Gyrokinetic projection of the divertor heat-flux width from present tokamaks to ITER. Nucl. Fusion 57 (11), 116023.

Chang, C. S., Churchill, M., Hager, R., Ku, S., Maingi, R., Menard, J., Loarte, A., Pitts, R., Parail, V., Romanelli, M. \& Köchl, F. 2018 Wide divertor heat-flux width in ITER from self-organization between the neoclassical and turbulent transports across the separatrix surface. In 27th IAEA Fusion Energy Conference, CN-258. Available at: https://conferences.iaea. org/event/151/contributions/6356/.

Coenen, J. W., Arnoux, G., Bazylev, B., Matthews, G. F., Autricque, A., Balboa, I., Clever, M., Dejarnac, R., Coffey, I., Corre, Y., et al. 2015 ELM-induced transient tungsten melting in the JET divertor. Nucl. Fusion 55 (2), 023010.

Courtois, X., Aumeunier, M., Balorin, C., Migozzi, J. B., Houry, M., Blanckaert, K., Moudden, Y., Pocheau, C., Saille, A., Hugot, E., et al. 2019 Full coverage infrared thermography diagnostic for WEST machine protection. Fusion Engng Des. 146, 2015-2020.

Creely, A. J., Greenwald, M. J., Ballinger, S. B., Brunner, D., Canik, J., Doody, J., FülöP, T., Garnier, D. T., Granetz, R., Gray, T. K., et al. Overview of the SPARC tokamak. J. Plasma Phys. 86. doi:10.1017/S0022377820001257.

De Temmerman, G., Kirk, A., Nardon, E., Tamain, P., \& MaSt Team. 2011 Heat load asymmetries in MAST. J. Nucl. Mater. 415 (1), S383-S386.

Dudson, B. 2019. FreeGS: Free boundary Grad-Shafranov solver. Github [Software: source code, version 0.3.0]. Available at: https://github.com/bendudson/freegs.

Eich, T., Leonard, A. W., Pitts, R. A., Fundamenski, W., Goldston, R. J., Gray, T. K., Herrmann, A., KirK, A., Kallenbach, A., Kardaun, O., et al. 2013 Scaling of the tokamak near the scrape-off layer H-mode power width and implications for ITER. Nucl. Fusion 53 (9), 093031.

Eich, T., Manz, P., Goldston, R. J., Hennequin, P., David, P., Faitsch, M., Kurzan, B., Sieglin, B. \& Wolfrum, E. 2020 Turbulence driven widening of the near-SOL power width in ASDEX upgrade H-mode discharges. Nucl. Fusion 60 (5), 056016.

Eich, T., Sieglin, B., Scarabosio, A., Fundamenski, W., Goldston, R.J., Herrmann, A. \& (ASDEX UpgRAdE TEAM) 2011a. Inter-ELM power decay length for JET and ASDEX upgrade: measurement and comparison with heuristic drift-based model. Phys. Rev. Lett. 107 (21), 215001.

Eich, T., Sieglin, B., Thornton, A. J., Faitsch, M., Kirk, A., Herrmann, A. \& Suttrop, W. 2017 ELM divertor peak energy fluence scaling to ITER with data from JET, MAST and ASDEX upgrade. Nucl. Mater. Energy 12, 84-90.

Eich, T.G., Thomsen, H., Fundamenski, W., Arnoux, G., Brezinsek, S., Devaux, S., HerRmann, A., JACHMich, S., RAPP, J., \& JET-EFDA CONTRIBUtORS. 2011b Type-I ELM power deposition profile width and temporal shape in JET. J. Nucl. Mater. 415 (1), S856-S859.

Garzotti, L., Challis, C., Dumont, R., Frigione, D., Graves, J., Lerche, E., Mailloux, J., MAntsinen, M., Rimini, F., CASSON, F., et al. 2019 Scenario development for D-T operation at JET. Nucl. Fusion 59 (7), 076037.

Goldston, R. J. 2011 Heuristic drift-based model of the power scrape-off width in low-gas-puff H-mode tokamaks. Nucl. Fusion 52 (1), 013009. 
Goldston, R. J., Reinke, M. L. \& Schwartz, J. A. 2017 A new scaling for divertor detachment. Plasma Phys. Control. Fusion 59 (5), 055015.

Guillemaut, C., Lennholm, M., Harrison, J., Carvalho, I., Valcarcel, D., Felton, R., Griph, S., Hogben, C., Lucock, R., Matthews, G. F., et al. 2017 Real-time control of divertor detachment in H-mode with impurity seeding using Langmuir probe feedback in JET-ITER-like wall. Plasma Phys. Control. Fusion 59 (4), 045001.

Hender, T. C., Wesley, J. C., Bialek, J., Bondeson, A., Boozer, A. H., Buttery, R. J., Garofalo, A., Goodman, T. P., Granetz, R. S., Gribov, Y., et al. 2007 MHD stability, operational limits and disruptions. Nucl. Fusion 47 (6), S128.

Henderson, S. S., Bernert, M., Brezinsek, S., Carr, M., Cavedon, M., Dux, R., Gahle, D. S., Harrison, J., Kallenbach, A., Lipschultz, B., et al. 2019 An assessment of nitrogen concentrations from spectroscopic measurements in the JET and ASDEX upgrade divertor. Nucl. Mater. Energy 18, 147-152.

Hirai, T., Pintsuk, G., Linke, J. \& Batilliot, M. 2009 Cracking failure study of ITER-reference tungsten grade under single pulse thermal shock loads at elevated temperatures. J. Nucl. Mater. $390,751-754$.

Hughes, J. W., Snyder, P. B., Reinke, M. L., LaBombard, B., Mordijck, S., Scott, S., Tolman, E., BAeK, S. G., Golfinopoulos, T., Granetz, R. S., et al. 2018 Access to pedestal pressure relevant to burning plasmas on the high magnetic field tokamak Alcator C-Mod. Nucl. Fusion 58 (11), 112003.

Hughes, J. W., Howard, N. T., Rodriguez-Fernandez, P., Creely, A. J., Kuang, A. Q., Snyder, P. B., Wilks, T. M., Sweeney, R., Greenwald, M. \& The SPARC Team 2020 High confinement access and edge pedestal structure in the SPARC tokamak. J. Plasma Phys. (under review).

HUlSE, R. A. 1983 Numerical studies of impurities in fusion plasmas. Nucl. Tech.-Fusion 3 (2), 259-272.

InCropera, F. P., DeWitt, D. P., Bergman, T. L. \& LaVine, A. S. 2007 Fundamentals of Heat and Mass Transfer. Wiley.

Kallenbach, A., Bernert, M., Beurskens, M., Casali, L., Dunne, M., Eich, T., Giannone, L., Herrmann, A., MarascheK, M., Potzel, S., et al. 2015 Partial detachment of high power discharges in ASDEX upgrade. Nucl. Fusion 55 (5), 053026.

Kallenbach, A., Bernert, M., Eich, T., Fuchs, J. C., Giannone, L., Herrmann, A., SCHWEInZER, J. \& TREUTTERER, W. 2012 Optimized tokamak power exhaust with double radiative feedback in ASDEX upgrade. Nucl. Fusion 52 (12), 122003.

Katramados, I., Fishpool, G., Fursdon, M., Whitfield, G., Thompson, V. \& Meyer, H. 2011 MAST upgrade closed pumped divertor design and analysis. Fusion Engng Des. 86 (9-11), 1595-1598.

Kuang, A. Q., Cao, N. M., Creely, A. J., Dennett, C. A., Hecla, J., LaBombard, B., Tinguely, R. A., Tolman, E. A., Hoffman, H., Major, M., et al. 2018 Conceptual design study for heat exhaust management in the ARC fusion pilot plant. Fusion Engng Des. 137, 221-242.

Kunushinin, A. S., Janeschitz, G., Loarte, A., Pacher, H. D., Coster, D., Reiter, D. \& SCHNEIDER, R. 2001 Critical issues in divertor optimisation for ITER-FEAT. J. Nucl. Mater. 290, 887-891.

Kunushinin, A. S., Pacher, H. D., Pacher, G. W., Kotov, V., Pitts, R. A. \& Reiter, D. 2013 Consequences of a reduction of the upstream power SOL width in ITER. J. Nucl. Mater. 438, S203-S207.

LaBombard, B., Marmar, E., Irby, J., Terry, J. L., Vieira, R., Wallace, G., Whyte, D. G., Wolfe, S., WuKITCH, S., BAEK, S., et al. 2015 ADX: a high field, high power density, advanced divertor and RF tokamak. Nucl. Fusion 55 (5), 053020.

Lipschultz, B., Whyte, D. G., Granetz, R. S., Loarte, A., Reinke, M. L. \& Wolfe, S. M. 2011 The scaling of fuel recovered following un-mitigated disruptions in Alcator C-Mod with high-Z PFCs. J. Nucl. Mater. 415 (1), S813-S816.

Loarte, A., Lipschultz, B., Kukushinin, A. S., Matthews, G. F., Stangeby, P. C., Asakura, N., Counsell, G. F., Federici, G., Kallenbach, A., Krieger, K., et al. 2007 Power and particle control. Nucl. Fusion 47 (6), S203. 
Lunt, T., Zohm, H., Herrmann, A., Kallenbach, A., Dunne, M., Feng, Y., Neu, R. \& WiSCHMEIER, M. 2017 Proposal of an alternative upper divertor in ASDEX upgrade supported by EMC3-EIRENE simulations. Nucl. Mater. Energy 12, 1037-1042.

Marmar, E.S. \& Alcator C-Mod Group. 2007 The alcator c-mod program. Fusion Sci. Technol. 51 (3), 261-265.

Pintsuk, G., KÜHnlein, W., Linke, J. \& Rödig, M. 2007 Investigation of tungsten and beryllium behaviour under short transient events. Fusion Engng Des. 82 (15-24), 1720-1729.

Pitts, R. A., Bardin, S., Bazylev, B., van den Berg, M. A., Bunting, P., CarpentierChouchana, S., Coenen, J. W., Dejarnac, R., Escourbiac, F., et al. 2017 Physics conclusions in support of ITER W divertor monoblock shaping. Nucl. Mater. Energy 12, 60-74.

Pitts, R. A., Bonnin, X., Escourbiac, F., Frerichs, H., Gunn, J. P., Hirai, T., Kukushkin, A. S., Kaveeva, E., Miller, M. A., Moulton, D., et al. 2019 Physics basis for the first ITER tungsten divertor. Nucl. Mater. Energy 20, 100696.

Reimerdes, H., Alberti, S., Blanchard, P., Bruzzone, P., Chavan, R., Coda, S., Duval, B. P., Fasoli, A., LABit, B., Lipschultz, B., et al. 2017 TCV divertor upgrade for alternative magnetic configurations. Nucl. Mater. Energy 12, 1106-1111.

REINKE, M. L. 2017 Heat flux mitigation by impurity seeding in high-field tokamaks. Nucl. Fusion 57 (3), 034004.

Reinke, M. L., Brunner, D., Golfinopoulos, T., Hubbard, A. E., Hughes, J. W., Kuang, A. Q., LaBombard, B., Marmar, E., Mumgaard, R., Terry, J. L., et al. 2019 Radiative heat exhaust in Alcator C-Mod I-mode plasmas. Nucl. Fusion 59 (4), 046018.

RicCARDO, V. \& LOARTE, A. 2005 Timescale and magnitude of plasma thermal energy loss before and during disruptions in JET. Nucl. Fusion 45 (11), 1427.

Rodriguez-Fernandez, P., Howard, N. T., Greenwald, M. J., Creely, A. J., Hughes, J. W., Wright, J. C., Holland, C., Lin, Y., Sciortino, F. \& The SPARC Team 2020 Predictions of core plasma performance for the SPARC tokamak. J. Plasma Phys. 86. doi:10.1017/ S0022377820001075.

Rognlien, T. D., Milovich, J. L., Rensink, M. E. \& Porter, G. D. 1992 A fully implicit, time dependent 2-D fluid code for modeling tokamak edge plasmas. J. Nucl. Mater. 196-198, 347-351.

Silburn, S. A., Matthews, G. F., Challis, C. D., Frigione, D., Graves, J. P., Mantsinen, M. J., Belonohy, E., Hobirk, J., Iglesias, D., Keeling, D. L., et al. 2017 Mitigation of divertor heat loads by strike point sweeping in high power JET discharges. Phys. Scr. 2017 (T170), 014040 .

SKINNER, C. H. \& GiAnfranco, F. 2006 Is carbon a realistic choice for ITER's divertor? Phys. Scr. 2006 (T124), 18.

Sorbom, B. N., Ball, J., Palmer, T. R., Mangiarotti, F. J., Sierchio, J. M., Bonoli, P., Kasten, C., Sutherland, D. A., Barnard, H. S., HaAkonsen, C. B., et al. 2015 ARC: a compact, high-field, fusion nuclear science facility and demonstration power plant with demountable magnets. Fusion Engng Des. 100, 378-405.

Stangeby, P. 2000 The Plasma Boundary of Magnetic Fusion Devices. CRC Press.

Streibl, B., Lang, P. T., Leuterer, F., Noterdaeme, J. M. \& StÄBler, A. 2003 Chapter 2: machine design, fueling, and heating in ASDEX upgrade. Fusion Sci. Technol. 44 (3), 578-592.

Sweeney, R., Creely, A. J., Doody, J., FÜlöP, T., Garnier, D. T., Granetz, R., Greenwald, M., Hesslow, L., IRBY, J., Izzo, V. A., et al. 2020 MHD stability and disruptions in the SPARC tokamak. J. Plasma Phys. 86. doi:10.1017/S0022377820001129.

Tanabe, T., Bekris, N., CoAd, P., Skinner, C. H., Glugla, M. \& Miya, N. 2003 Tritium retention of plasma facing components in tokamaks. J. Nucl. Mater. 313-316, 478-490.

Telesca, G., IVAnova-Stanik, I., Zagórski, R., Brezinsek, S., CZarnecka, A., Drewelow, P., Giroud, C., Huber, A., Wiesen, S., Wischmeier, M., et al. 2017 High power neon seeded JET discharges: experiments and simulations. Nucl. Mater. Energy 12, 882-886.

ULRICKSON, M., JET TEAM \& TFTR TEAM. 1990 A review of carbon blooms on JET and TFTR. J. Nucl. Mater. 176-177, 44-50.

Umansky, M. V., Krasheninnikov, S. I., LaBombard, B. \& Terry, J. L. 1998 Comments on particle and energy balance in the edge plasma of Alcator C-Mod. Phys. Plasmas 5 (9), 3373-3376. 
Umansky, M. V., LaBombard, B., Brunner, D., Golfinopoulos, T., Kuang, A. Q., Rensink, M. E., Terry, J. L., Wigram, M. \& Whyte, D. G. 2019 Study of passively stable, fully detached divertor plasma regimes attained in innovative long-legged divertor configurations. Nucl. Fusion 60 (1), 016004.

Umansky, M. V., LaBombard, B., Brunner, D., Rensink, M. E., Rognlien, T. D., Terry, J. L. \& WhYte, D. G. 2017 Attainment of a stable, fully detached plasma state in innovative divertor configurations. Phys. Plasmas 24 (5), 056112.

US DEPARTMENT OF ENERGY. 2015 Report on science challenges and research opportunities in plasma materials interactions. From the US DoE fusion energy sciences workshop on plasma materials interactions, May 4-7, 2015. Available at: https://www.burningplasma.org/resources/ref/ Workshops2015/PMI/PMI_fullreport_21_Aug2015.pdf

Wiesen, S., Reiter, D., Kotov, V., Baelmans, M., Dekeyser, W., Kukushin, A. S., Lisgo, S. W., Pitts, R. A., Rozhansky, V., Saibene, G., et al. 2015 The new SOlPS-ITER code package. J. Nucl. Mater. 463, 480-484.

Wischmeier, M., The ASDEX Upgrade Team \& JET EFDA Contributors. 2015 High density operation for reactor-relevant power exhaust. J. Nucl. Mater. 463, 22-29. 